\title{
Syopsis of the
}

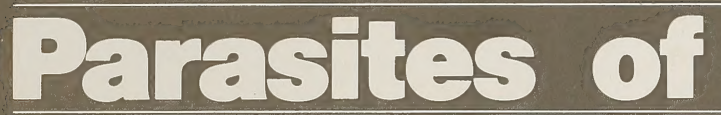

Domestioated Mennmals

of Canada

Murray J. Kennedy

and

Robert A. Newman 
Copies of this publication are available from the Queen's Printer, 11510 Kingsway Avenue, Edmonton T5G 2Y5 Orders must be accompanied by a cheque for $\$ 5.00$ made out to the Provincial Treasurer. 


\section{Synopsis of the}

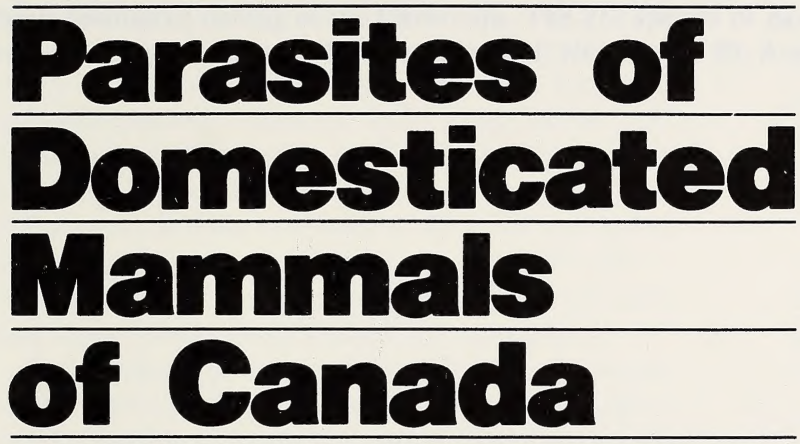

Murray J. Kennedy and

Robert A. Newman

Alberta Agriculture

Veterinary Laboratory

6909 - 116 Street,

Edmonton, Alberta

Canada

T6H 4P2 



\begin{abstract}
This synopsis contains Parasite-Host and Host-Parasite lists of 212 species of parasites from seven domesticated animals. Four species, the goat (Capra hircus), ox (Bos taurus), pig (Sus scrofa), and sheep (Ovis aries) belong in the Artiodactyla; one species, the horse (Equus caballus) in the Perissodactyla; and two species, the cat (Felis catus) and dog (Canis familiaris) belong in the Carnivora. The 212 species of parasites are distributed among higher taxa as follows: Protozoa - 36; Trematoda - 12; Cestoidea - 21; Nematoda - 95; Acanthocephala - 2; Chelicerata - 15; and Uniramia - 31.
\end{abstract}





\section{Introduction}

The compiled information covers the period from 1877 to December 1985 and was extracted from numerous publications in the primary scientific literature. Journal series such as the Canadian Journal of Zoology, Canadian Journal of Research, Canadian Veterinary Journal, Journal of Protozoology, Proceeding of the Helminthological Society of Washington, and the Journal of Parasitology were searched in their entirety. Abstracting series such as Zoological Records, Helminthological Abstracts, Protozoology Abstracts, and Veterinary Bulletin were scanned for additional references. Theses and reports of federal and provincial governments and of other institutions were not searched extensively, hence, some existing records may have been omitted.

In providing references to the parasite-host-distribution data, we have included only reports based on original records, but screening all reports to ensure complete exclusion of repetitive records based on the same material was not possible. Publications that do not provide original parasitehost-distribution records but deal with some aspect of Canadian veterinary parasitology are compiled in a supplementary list of references.

The synopsis indicates that up to December, 1985, at least 212 species of parasites have been reported from seven species of domesticated animals. Parasites not identified to the species level are not included in the total number. The 212 species are divided among major taxa as follows: Protozoa -36; Trematoda - 12; Cestoidea - 21; Nematoda -95; Acanthocephala - 2, Chelicereta - 15; and Uniramia - 31 .

The taxonomic arrangement adopted for each phylum or class of parasitic organism included in Canadian records, in most cases, is as follows:

Protozoa - Levine et al. (1980)
Trematoda - Brooks et al. (1985)
Cestoidea - Schmidt (1986)
Nematoda - Anderson et al. (1974-1983)
Acanthocephala - Amin (1982)
Acarina - Krantz (1978)
Diptera - Arnett et al. (1980)
Mallophaga - Emerson (1972)
Siphonaptera - Holland (1984)
Siphunculata - Strojanovich and Pratt (1965)

Host nomenclature and classification conforms to that of Novak and Paradiso (1983). The entries in the synopsis are organized in accordance with the format used by Margolis and Arthur (1979) in their Synopsis of the parasites of fishes of Canada.

The Parasite-Host List is organized by higher taxa of parasites in the following order: Protozoa, Platyhelminthes (Trematoda, Cestoidea), Nemathelminthes (Nematoda), Acanthocephala, Chelicerata and Uniramia. Species, genera, and family names are listed in alphabetical order within their respective next higher taxon, which in the case of a family is either a superfamily or an order, depending on the group in question and the usefulness of including the superfamily or order category.
For each parasite species listed, the following information is provided:

1) The current scientific name, including author(s) and date(s), followed by any recognized synonyms that may have been used in establishing the Canadian record(s). No attempt has been made to evaluate systematically the validity of published records, but attention is drawn to obvious errors. Adult and larval stages are recorded separately for the Cestoidea and Nematoda.

2) The site of occurrence of the parasite in its host(s) (aberrant sites usually are not included). When the site was not given, the likely site was determined from other records, and is placed in square brackets.

3) The hosts. Numbers in parentheses after each host name correspond with the numbers assigned to the references establishing the particular parasite-host records.

4) Distribution (Dist.) Species distribution is indicated by Provincial or Territory boundaries listed in alphabetical order as follows: Alta (Alberta), BC (British Columbia), Man (Manitoba), NB (New Brunswick), Nfld (Newfoundland), NS (Nova Scotia), NWT (Northwest Territories), Ont (Ontario), PEI (Prince Edward Island), Que (Quebec), Sask (Saskatchewan), and YT (Yukon Territory).

5) Authors responsible for the published records are listed in chronological order. To enable the reader to grasp at a glance the author(s) responsible for particular parasitehost-locality records the references are numbered and listed under "Records" and the corresponding number(s) placed after the host name. Each reference is followed by the locality or localities from which the parasite was (were) reported. When only one host is listed for a particular parasite, the references are not numbered, and when all records are from the same locality, the latter was not listed after the authors' names.

6) Under remarks, explanatory comments, as required, are offered on systematics, nomenclature, misidentifications, and synonyms of parasites; and on the hosts, sites within hosts, and validity of previous records.

In the Host-Parasite List, within each order, the families, genera, and species are listed alphabetically. After the name of each parasite species, its geographic distribution in the host in question is given in parentheses.

The REFERENCES are given in two parts, the first includes those directly referred to in the text, and the second, SUPPLEMENTARY REFERENCES, contains a list of publications dealing with various aspects of parasites of Canadian domestic mammals but which do not contain original parasite-host records.

To facilitate access to information in the parasite-host and host-parasite lists, the publication concludes with an INDEX to parasite and host scientific names. 



\section{PARASITE-HOST LIST}


Digitized by the Internet Archive in 2015 


\section{KINGDOM ANIMALIA \\ SUBKINGDOM PROTOZOA}

\section{PHYLUM APICOMPLEXA}

\section{CLASS SPOROZOEA}

ORDER EUCOCCIDIIDA

\section{SUBORDER ADELEINA}

Klossiella equi Baumann, 1946

Site: kidney

Host: Equus caballus

Dist.: NB

Record: Austin and Dies 1981

\section{SUBORDER EIMERIINA}

Cryptosporidium spp.

Site: intestine

Host: Bos taurus (1)

Equus caballus (2)

Dist.: Ont, Sask

Record: 1. Sanford and Josephson 1982 (Ont);

2. Gajadhar et al. 1985 (Sask)

Eimeria ahsata Honess, 1942

Site: [intestine]

Host: Ovis aries

Dist.: Alta

Record: Mahrt 1969

Eimeria alabamensis Christensen, 1941

Site: [intestine]

Host: Bos taurus

Dist.: Ont

Record: Slocombe 1973a

Eimeria arloingi (Marotel, 1905) Martin, 1909

Site: [intestine]

Hosts: Bos taurus (2)

Ovis aries $(1,2)$

Dist.: Alta, Ont

Records: 1. Mahrt 1969 (Alta); 2. Slocombe 1973a (Ont)

Eimeria aubernensis Christensen and Porter, 1939

Site: [intestine]

Host: Bos taurus

Dist.: Ont

Record: Slocombe 1973a

Eimeria bovis (Zublin, 1908) Fiebiger, 1912

Site: [intestine]

Host: Bos taurus

Dist.: Ont

Record: Slocombe 1973a

Eimeria bukidnonensis Tubangui, 1931

Site: [intestine]

Host: Bos taurus

Dist.: Ont

Record: Slocombe 1973a
Eimeria canadensis Bruce, 1921

Site: [intestine]

Host: Bos taurus

Dist.: Ont

Record: Slocombe 1973a

Eimeria canis Wenyon, 1923

Site: [intestine]

Host:Canis familiaris

Dist.: Ont, Que

Records: Choquette and Gelinas 1950 (Que);

Slocombe 1973a (Ont)

Eimeria crandallis Honess, 1942

Site: [intestine]

Hosts: Equus caballus (2)

Ovis aries (1)

Dist.: Alta, Ont

Records: 1. Mahrt 1969 (Alta); 2. Slocombe 1973a (Ont)

Eimeria cylindrica Wilson, 1931

Site: [intestine]

Host: Bos taurus

Dist.: Ont

Record: Slocombe 1973a

Eimeria debliecki Douwes, 1921

Site: [intestine]

Host: Sus scrofa

Dist.: Ont

Record: Slocombe 1973a

Eimeria ellipsoidalis Becker and Frye, 1929

Site: [intestine]

Host: Bos taurus

Dist.: Ont

Record: Slocombe 1973a

Eimeria faurei (Moussu and Marotel, 1902) Martin, 1909

Site: [intestine]

Host: Ovis aries

Dist.: Alta

Record: Mahrt 1969

Eimeria granulosa Christensen, 1938

Site: [intestine]

Hosts: Equus caballus (2) Ovis aries (1)

Dist.: Alta, Ont

Records: 1. Mahrt 1969 (Alta); 2. Slocombe 1973a (Ont)

Eimeria intricata Spiegl, 1925

Site: [intestine]

Hosts: Equus caballus (2) Ovis aries (1)

Dist.: Alta, Ont

Records: 1. Mahrt 1969 (Alta); 2. Slocombe 1973a (Ont) 
Eimeria leuckarti (Flesch, 1883) Reichenow, 1940

Site: [intestine]

Host: Equus caballus

Dist.: Ont

Record: Slocombe 1973a

Eimeria ninakohlyakimovae Yakimoff and

Site: [intestine]

Rastegaieff, 1930

Host: Ovis aries

Dist.: Alta, Ont

Records: Mahrt 1969 (Alta); Slocombe 1973a

(Ont)

Eimeria pallida Christensen, 1938

Site: [intestine]

Host: Ovis aries

Dist.: Alta

Record: Mahrt 1969

Eimeria parva Kotlan, Mocsy, and Vajda, 1929

Site: [intestine]

Host: Ovis aries

Dist.: Alta, Ont

Records: Mahrt 1969 (Alta); Slocombe 1973a

(Ont)

Eimeria punctata Landers, 1955

Site: [intestine]

Host:Ovis aries

Dist.: Alta

Record: Mahrt 1969

Eimeria wyomingensis Huizinga and Winger, 1942

Site: [intestine]

Host: Bos taurus

Dist.: Ont

Record: Slocombe 1973a

Eimeria zuernii (Rivolta, 1878) Martin, 1909

Site: [intestine]

Host: Bos taurus

Dist.: Ont

Record: Slocombe 1973a

Eimeria spp.

Site: [intestine]

Host: Ovis aries

Dist.: Que

Record: Swales 1940b

Isospora bigemina (Stiles, 1891) Luhe, 1906

Site: intestine

Host: Canis familiaris

Dist.: Que

Record: Choquette and Gelinas 1950

Isospora felis Wenyon, 1926

Site: [intestine]

Hosts: Canis familiaris $(1,2)$

Felis catus (2)

Dist.: Ont, Que

Records: 1. Choquette and Gelinas 1950 (Que);

2. Slocombe 1973a (Ont)
Isospora rivolta (Grassi, 1879) Wenyon, 1923

Site: [intestine]

Hosts: Canis familiaris $(1,2)$

Felis catus (2)

Dist.: Ont, Que

Records: 1. Choquette and Gelinas 1950 (Que);

2. Slocombe 1973a (Ont)

Isospora spp.

Site: [intestine]

Hosts: Canis familiaris $(1,2,3,4,5)$

Felis catus (5)

Dist.: Alta, NB, NWT, Que, Sask

Records: 1. Saunders 1949 (Alta); 2. Unruh et al. 1973 (Alta, NWT, Sask); 3. Anvik et al. 1974 (Sask); 4. Seah et al. 1975 (Que); 5. Malloy and Embil 1978 (NB)

Remarks: Saunders (1949) believed his species was probably I. bigemina.

Sarcocystis spp.

Site: heart

Hosts: Bos taurus (1)

Equus caballus (1)

Ovis aries (2)

Dist.: Ont, Sask

Records: 1. Slocombe 1973a(Ont); 2. Moteane et al. 1979 (Sask)

Toxoplasma gondii Nicolle and Manceaux, 1908

Site: unspecified

Hosts: Canis familiaris

Dist.: Ont Felis catus

Record: Hulland 1956

Toxoplasma spp.

Site: brain, spinal cord

Hosts: Canis familiaris (1)

Equus caballus $(1,2)$

Felis catus (1)

Ovis aries (1)

Sus scrofa (l)

Dist.: Ont, Sask

Records: 1. Slocombe 1973a (Ont); 2. Clark et al. 1981 (Sask)

Unidentified Eucoccidiida

Site: [intestine]

Hosts: Bos taurus (2)

Ovis aries $(1,3)$

Dist.: Ont, Que, Sask

Records: 1. Griffiths 1937 (Que); 2. Slocombe

1973a (Ont); 3. Monteane et al. 1979 (Sask)

PHYLUM CILIOPHORA

CLASS KINETOFRAGMINOPHOREA

ORDER TRICHOSTOMATIDA

Balantidium spp.

Site: [intestine]

Host: Sus scrofa

Dist.: Ont

Record: Slocombe 1973a 
SUBPHYLUM MASTIGOPHORA

CLASS ZOOMASTIGOPHOREA

ORDER DIPLOMONADIDA

FAMILY HEXAMITIDAE

Giardia canis Hegner, 1922

Site: [intestine]

Host: Canis familiaris

Dist.: Que

Records: Choquette and Gelinas 1950

Giardia spp.

Site: [intestine]

Host: Canis familiaris

Dist.: Ont

Records: Slocombe 1973a

\section{ORDER KINETOPLASTIDA}

Trypanosoma equiperdum Doflein, 1901

Site: blood

Host: Equus caballus

Dist.: Alta

Record: Watson and Hadwen 1912

Trypanosoma rutherfordi Watson and Hadwen, 1912

Site: blood

Host: Bos taurus

Dist.: BC

Record: Watson and Hadwen 1912

Trypanosoma theileri Laveran, 1902

Site: blood

Host: Bos taurus

Dist.: Ont

Record: Woo et al. 1970

Trypanosoma uniforme Bruce et al., 1911

Site: blood

Host: Bos taurus

Dist.: Ont

Record: Woo et al. 1970

Remarks: Woo et al. (1970) stated only that the Trypanosome resembled T. uniforme.

Trypanosoma spp.

Site: blood

Host: Bos taurus

Dist.: BC

Record: Watson and Hadwen 1912
Trichomonas spp.

Site: intestine

Hosts: Bos taurus (2)

Canis familiaris (1)

Equus caballus (2)

Dist.: Ont

Sus scrofa (2)

Records: 1. Comfort and Axelson 1962; 2.

Slocombe 1973a

SUBPHYLUM SARCODINA

CLASS LOBOSEA

ORDER AMOEBIDA

Endolimax nana (Wenyon and O'Conner, 1917) Brug, 1918

Site: [intestine]

Host: Canis familiaris

Dist.: Sask

Record: Unruh et al. 1973

Entamoeba blattae

Site: [intestine]

Host: Canis familiaris

Dist.: Que

Record: Choquette and Gelinas 1950

Remarks: Choquette and Gelinas (1950)

considered $C$. familiaris an unnatural host for $E$. blattae. This protozoan is normally a parasite of cockroaches and termites.

Entamoeba coli (Grassi, 1879) Casagrandi and

Site: [intestine] Barbagallo, 1895

Host: Canis familiaris

Dist.: Que, Sask

Records: Unruh et al. 1973 (Sask); Seah et al. 1975 (Que)

Entamoeba histolytica Schaudinn, 1903

Site: [intestine]

Host: Canis familiaris

Dist.: Que, Sask

Records: Unruh et al. 1973 (Sask); Wittnich 1976 (Que) 


\section{PHYLUM PLATYHELMINTHES}

\section{CLASS TREMATODA}

\section{SUBCLASS DIGENEA}

\section{FAMILY DICROCOELIIDAE}

Dicrocoelium dendriticum (Rudolphi, 1819)

Looss, 1899

Syn.: Dicrocoelium lanceolatum Stiles and Hassal, 1898

Site: bile ducts

Hosts: Bos taurus $(5,6)$

Ovis aries (1 Que,2,3,4)

Dist.: BC, Nfld, NS, PEI, Que

Records: 1. Conklin and Baker 1930 (NS, PEI, Que); 2. Swales 1933a (Que), 3. 1940a (NS); 4. Smith and Archibald 1965 (NS); 5. Smith and Threlfall 1973 (Nfld); 6. Lewis 1974 (BC)

\section{FAMILY DIPLOSTOMIDAE}

Alaria arisaemoides Augustine and Uribe, 1927

Site: intestine

Host: Canis familiaris

Dist.: Sask

Record: Allen and Mills 1971

Alaria canis LaRue and Fallis, 1934

Site: [intestine]

Host: Canis familiaris

Dist.: Ont

Record: LaRue and Fallis 1934

Alaria spp.

Site: [intestine]

Host: Canis familiaris

Dist.: Alta, NWT, Ont, Que, Sask

Records: 1. Cameron et al. 1940 (NWT, Ont, Que, Sask); 2. Allen and Mills 1971 (Sask); 3. Unruh et al. 1973 (Alta, NWT, Sask); 4. Anvik et al. 1974 (Sask)

\section{FAMILY FASCIOLIDAE}

Fasciola hepatica Linnaeus, 1758

Site: [liver]

Host: Ovis aries

Dist.: Canada

Record: Swales 1940a

Fascioloides magna (Bassi, 1875) Ward, 1917

Site: liver

Host: Bos taurus

Dist.: Alta, Ont

Records: Swales 1933b (Alta); Slocombe 1973a (Ont)

\section{FAMILY HETEROPHYIDAE}

Cryptocotyle lingua (Creplin, 1825) Luhe, 1899

Site: [intestine]

Host: Canis familiaris

Dist.: Nfld, Que

Record: Cameron et al. 1940

\section{FAMILY OPISTHORCHIIDAE}

Metorchis conjunctus (Cobbold, 1860) Looss, 1899

Site: bile ducts, pancreatic duct

Hosts: Canis familiaris $(1,2,4,5,6,7)$

Felis catus $(3,7)$

Dist.: Alta, Man, NWT, Ont, Que, Sask

Records: 1. Cameron et al. 1940 (Man, Ont,

Que, Sask); 2. Mongeau 1961 (Man); 3.

Axelson 1962 (Ont); 4. McLeod 1967 (Man); 5. Unruh et al. 1973 (Alta, NWT, Sask); 6. Anvik et al. 1974 (Sask); 7. Watson 1981 exp. (Que)

Metorchis spp.

Site: [bile ducts]

Host: Canis familiaris

Dist.: Sask

Record: Allen and Mills 1971

Parametorchis complexus (Stiles and Hassall, 1894) Skrjabin, 1913

Syn.: Parametorchis manitobensis (Allen and Wardle, 1934) Kennedy, 1983

Site: bile ducts, gallbladder

Hosts: Canis familiaris (1) Felis catus (2)

Dist.: Alta, Man

Records: 1. Allen and Wardle 1934 (Man); 2.

Kennedy 1983 (Alta)

\section{FAMILY PARAMPHISTOMIDAE}

Calicophoron microbothrioides (Price and McIntosh, 1944) Eduardo, 1983

Syn.: Paramphistomum microbothrioides Price and McIntosh, 1944

Site: rumen

Host: Bos taurus

Dist.: Que

Record: Bouvry and Rau 1984

Paramphistomum liorchis Fischoeder, 1901

Site: rumen

Host: Bos taurus

Dist.: Que

Record: Bouvry and Rau 1984

Paramphistomum spp.

Site: [intestine]

Host: Bos taurus

Dist.: Alta, Ont

Record: Swales 1933b 


\section{FAMILY PLAGIORCHIIDAE}

Plagiorchis massino Petrov and Tikhonov, 1927

Site: [intestine]

Host: Felis catus

Dist.: Nfld

Record: Smith and Threlfall 1973

\section{FAMILY TROGLOTREMATIDAE}

Paragonimus kellicotti Ward, 1908

Site: lungs

Host: Canis familiaris

Dist.: Ont

Records: Nielsen 1955; Comfort and Axelson 1962; Gillick 1972

Remarks: Nielsen (1955) presumed his specimen was $P$. kellicotti.

Paragonimus spp.

Site: lungs

Hosts: Canis familiaris

Dist.: Ont

Sus scrofa

Record: Slocombe 1973a

Unidentified Digenea gen. spp.

Site: [intestine]

Host: Canis familiaris

Dist.: Man, NWT, Que

Records: Cameron et al. 1940 (Man, NWT,

Que); Seah et al. 1975 (Que); Ghadirian et al. 1976 (Que)

\section{CLASS CESTOIDEA}

\section{SUBCLASS EUCESTODA}

\section{ORDER CYCLOPHYLLIDEA}

\section{FAMILY ANOPLOCEPHALIDAE}

Anoplocephala magna (Abildgaard, 1789)

Site: [intestine]

$$
\text { Spengel, } 1905
$$

Host: Equus caballus

Dist.: Ont, Que

Records: Swales 1933b (Que); Slocombe 1973a

(Ont)

Anoplocephala perfoliata (Goeze, 1782)

Blanchard, 1848

Site: [intestine]

Host: Equus caballus

Dist.: Nfld, Ont

Records: Swales 1933b (Ont); Slocombe 1973a

(Ont); Smith and Threlfall 1973 (Nfld)

Anoplocephala spp.

Site: [intestine]

Host: Equus caballus

Dist.: Ont

Records: Swales 1933a; Slocombe 1973a
Moniezia benedeni (Moniez, 1879) Blanchard, 1891

Site: intestine

Host: Bos taurus

Dist.: NB, Que

Records: Swales 1933b (Que); Smith 1970 (NB)

Moniezia expansa (Rudolphi, 1810) Blanchard, 1891

Site: intestine

Hosts: Bos taurus (2 Que)

Ovis aries $(1,2,3,4,5,6,7,8,9,10,11)$

Dist.: Canada, Nfld, NS, Ont, Que

Records: 1. Swales 1933a (unspecified), 2.

1933b (Ont, Que); 3. Griffiths 1937 (Que); 4.

Fallis 1938 (Ont); 5. Swales 1939 (Que), 6.

1940a (Canada), 7. 1940b (Que); 8. Swales and Choquette 1940 (Que); 9. Swales et al. 1942

(Ont); 10. Smith and Archibald 1965 (NS); 11.

Smith and Threlfall 1973 (Nfld)

Moniezia spp.

Site: [intestine]

Hosts: Bos taurus $(1,2,3)$

Ovis aries $(4,5)$

Dist.: Nfld, Ont, Que, Sask

Records: 1. McGregor and Kingscote 1957

(Ont); 2. Frechette and Gibbs 1971 (Que); 3.

Slocombe 1973a (Ont); 4. Smith and Threlfall

1973 (Nfld); 5. Moteane et al. 1979 (Sask)

Paranoplocephala mamillana (Mehlis, 1831)

Baer, 1927

Syn.: Anoplocephala mamillana (Mehlis, 1831)

Blanchard, 1891

Site: [intestine]

Host: Equus caballus

Dist.: BC, Que, Sask

Record: Swales 1933b

Thysanosoma actinioides Diesing, 1835

Site: [intestine]

Host: Ovis aries

Dist.: Alta, BC, Canada, Sask

Records: Swales 1933a (BC), 1933b (Alta,

Sask), 1940a (Canada)

Thysanosoma spp.

Site: bile duct

Host: Ovis aries

Dist.: Ont, Sask

Records: Slocombe 1973a (Ont); Moteane et al. 1979 (Sask)

\section{FAMILY DILEPIDIDAE}

Dipylidium caninum (Linnaeus, 1758) Railliet, 1892

Site: [intestine]

Hosts: Canis familiaris (1 Ont,2,3,5,6,7)

Felis catus (1 Ont, 4)

Dist.: NB, Nfld, NWT, Ont, Que

Records: 1. Swales 1933b (Ont, Que); 2.

Cameron et al. 1940 (NWT, Ont); 3. Freeman

1962 (Ont); 4. Threlfall 1969 (Nfld); 5. Smith

and Threlfall 1973 (Nfld); 6. Unruh et al. 1973

(NWT); 7. Malloy and Embil 1978 (NB) 
Dipylidium spp.

Site: [intestine]

Hosts: Canis familiaris $(1,2)$ Felis catus (2)

Dist.: Ont, Sask

Records: 1. Allen and Mills 1971 (Sask); 2.

Slocombe 1973a (Ont)

\section{FAMILY TAENIIDAE}

Cysticercus bovis

Site: unspecified

Host: Bos taurus

Dist.: Ont, Sask

Records: Swales 1933b (Ont, Sask); Slocombe 1973a (Ont)

Cysticercus cellulosae

Site: liver

Host: Sus scrofa

Dist.: Man, Ont, Sask

Records: Osler and Clement 1883 (Ont); Swales 1933b (Man, Sask)

Cysticercus ovis Cobbold, 1869

Site: unspecified

Hosts: Ovis aries

Dist.: Canada, Man, NS

Records: Swales 1933b (Man), 1940a (Canada);

Soehl 1984 (NS)

Cysticercus tenuicollis Pallas, 1766

Site: unspecified

Hosts: Bos taurus (1 Ont, Que)

Ovis aries $(1,2,3)$

Dist.: Alta, Canada, Ont, Que

Records: 1. Swales 1933b (Alta, Ont, Que), 2.

1940a (Canada); 3. Slocombe 1973a (Ont)

Echinococcus granulosus (Batsch, 1786) Rudolphi, 1801

Site: intestine

Host: Canis familiaris

Dist.: Ont, Que

Records: Choquette 1956 exp. (Que); Slocombe 1973a (Ont)

Echinococcus granulosus (Batsch, 1786) Rudolphi,

Site: liver 1801 (hydatid cyst)

Host: Sus scrofa

Dist.: Ont

Record: Pallister and Plummer 1941 (Ont)

Echinococcus multilocularis Leuckart, 1863

Site: [intestine]

Host: Felis catus

Dist.: Sask

Record: Wobeser 1971

Echinococcus spp. (hydatid)

Site: liver

Host: Sus scrofa

Dist.: Ont

Record: Osler and Clement 1883
Multiceps spp.

Site: unspecified

Host: Sus scrofa

Dist.: Ont

Record: Slocombe 1973a

Taenia crassiceps (Zeder, 1800) Rudolphi, 1810

Site: [intestine]

Hosts: Canis familiaris (2)

Dist.: Ont

Canis familiaris $\mathrm{x}$ Canis latrans (1)

Records: 1. Freeman et al. 1961; 2. Freeman

1962 exp.

Taenia elliptica

Site: intestine

Host: Canis familiaris

Dist.: Que

Record: Osler 1877

Taenia hydatigena Pallas, 1766

Site: [intestine]

Host: Canis familiaris

Dist.: Nfld, Que

Records: Swales 1933b (Que); Sweatman and

Plummer 1957 exp. (Que); Threlfall 1969 (Nfld)

Taenia hydatigena Pallas, 1766 (cysticercus)

Site: mesenteries, peritoneum

Hosts: Ovis aries $(1,2)$

Sus scrofa (1)

Dist.: Que, Sask

Records: 1. Sweatman and Plummer 1957 exp.

(Que); 2. Moteane et al. 1979 (Sask)

Taenia pisiformis (Bloch, 1780) Gmelin, 1790

Site: [intestine]

Hosts: Canis familiaris $(1,3,4,5)$

C. familiaris $\mathrm{x}$ C. latrans (2)

Dist.: Alta, Nfld, NWT, Ont, Que

Records: 1. Swales 1933b (Que); 2. Freeman et al. 1961 (Ont); 3. Threlfall 1969 (Nfld); 4. Smith and Threlfall 1973 (Nfld); 5. Unruh et al. 1973 (Alta, NWT)

Taenia serialis (Gervais, 1847) Baillet, 1863

Site: [intestine]

Host: Canis familiaris

Dist.: Alta, NWT

Record: Unruh et al. 1973

Taenia taeniaeformis (Batsch, 1786) Wolffgel, 1863

Site: [intestine]

Host: Felis catus

Dist.: Nfld, Que

Records: Swales 1933b (Que); Smith and

Threlfall 1973 (Nfld)

Taenia spp.

Site: [intestine]

Hosts: Canis familiaris $(1,2,3)$

Felis catus (2)

Dist.: BC, Man, NWT, Ont, Que, Sask

Records: 1. Cameron 1940 (BC, Man, NWT, Ont, Que); 2. Slocombe 1973a (Ont); 3. Anvik et al. 1974 (Sask) 
Taenia/ Echinococcus spp. (egg)

Site: [intestine]

Host: Canis familiaris

Dist.: Alta, NWT, Sask

Records: Allen and Mills 1971 (Sask); Unruh et al. 1973 (Alta, NWT, Sask)

\section{ORDER PSEUDOPHYLLIDEA}

\section{FAMILY DIPHYLLOBOTHRIIDAE}

Diphyllobothrium dendriticum Nitsch, 1824

Site: [intestine]

Host: Felis catus

Dist.: Nfld

Records: Threlfall 1969; Smith and Threlfall 1973

Diphyllobothrium latum Luhe, 1910

Site: [intestine]

Host: Canis familiaris

Dist.: Alta, Nfld, NWT, Que, Sask

Records: Saunders 1949 (Alta); Threlfall 1969

(Nfld); Smith and Threlfall 1973 (Nfld); Unruh et al. 1973 (NWT); Anvik et al. 1974 (Sask);

Seah et al. 1975 (Que)

Diphyllobothrium spp.

Site: [intestine]

Hosts: Canis familiaris $(1,2,3,4,6)$ Felis catus (5)

Dist.: Alta, Man, Nfld, NWT, Ont, Que, Sask

Records: 1. Wardle and McColl 1937 (Ont); 2.

Cameron et al. 1940 (Alta, Man, Nfld, NWT,

Ont, Que, Sask); 3. Threlfall 1969 (Nfld); 4.

Allen and Mills 1971 (Sask); 5. Slocombe 1973a

(Ont); 6. Unruh et al. 1973 (Alta, NWT, Sask)

Unidentified Cestoidea gen. spp.

Site: Intestine

Host: Canis familiaris

Dist.: Man, Que

Records: Mongeau 1961 (Man); Seah et al.

1975 (Que)

\section{PHYLUM NEMATHELMINTHES}

CLASS NEMATODA

SUBCLASS ADENOPHOREA

ORDER ENOPLIDA

\section{SUPERFAMILY DIOCTOPHYMATOIDEA}

FAMILY DIOCTOPHYMATIDAE

Dioctophyma renale (Goeze, 1782) Collet-Meygret, 1802

Site: kidney

Host: Canis familiaris

Dist.: Man, NWT, Ont, Que

Records: Swales 1933b (Ont, Que); Cameron et al. 1940 (Man); McLeod 1967 (Man); Unruh et al. 1973 (NWT)

\section{SUPERFAMILY TRICHINELLOIDEA}

FAMILY TRICHINELLIDAE

Trichinella spiralis (Owen, 1835) Railliet, 1894 (cyst/larva)

Site: diaphragm

Host: Sus scrofa

Dist.: BC, Man, NB, NS, Ont, PEI, Que

Records: Osler and Clement 1883 (Ont);

Cameron 1938 (Man, Ont, PEI, Que), 1939

(Man, Ont, PEI, Que), 1940a (Que); Moynihan and Musfeldt 1949 (BC); Frank 1952 (NB, NS, PEI); Tailyour and Steele 1960 (BC)

\section{FAMILY TRICHURIDAE}

Capillaria aerophila Creplin, 1839

Site: [lungs]

Host: Canis familiaris

Dist.: Que

Record: Seah et al. 1975

Capillaria hepatica Bancroft, 1896

Site: liver

Hosts: Felis catus (1) Equus caballus (2)

Dist.: Alta, NB

Records: 1. Malloy and Embil 1978 (NB); 2.

Nation and Dies 1978 (Alta)

Capillaria longipes Ransom, 1911

Site: [intestine]

Host: Ovis aries

Dist.: Canada, Que

Records: Swales 1939 (Que), 1940a (Canada)

Capillaria spp.

Site: unspecified

Hosts: Bos taurus $(3,5)$

Canis familiaris (6)

Felis catus (6)

Ovis aries $(1,2,4)$

Dist.: NS, Ont, Que

Records: 1. Griffiths 1937 (Que); 2. Fallis 1938

(Ont); 3. McGregor and Kingscote 1957 (Ont);

4. Smith and Archibald 1965 (NS); 5. Frechette and Gibbs 1971 (Que); 6. Slocombe 1973a (Ont)

Trichuris ovis (Abildgaard, 1795) Smith, 1908

Site: [intestine]

Hosts: Bos taurus (1 Que,7)

Ovis aries $(1,2,3,4,5,6,7,8)$

Dist.: Canada, NB, Nfld, NS, Ont, Que

Records: 1. Swales 1933b (NS, Ont, Que); 2.

Fallis 1938 (Ont); 3. Swales 1940a (Canada), 4.

1940b (Que); 5. Swales and Choquette 1940

(Que); 6. Smith and Archibald 1965 (NS), 7.

1968 nat./exp. (NB); 8. Smith and Threlfall

1973 (Nfld) 
Trichuris suis (Shrank, 1788)

Site: [intestine]

Host: Sus scrofa

Dist.: Ont, Que

Records: Swales 1933b (Ont, Que); Martin et al. 1974 (Que)

Trichuris vulpis (Frohlich, 1789)

Site: [intestine]

Host: Canis familiaris

Dist.: Nfld, Ont, Que

Records: Osler 1877 (Que); Swales 1933b (Ont, Que); Cameron et al. 1940 (Ont); Choquette and Gelinas 1950 (Que); Smith and Threlfall 1973 (Nfld); Seah et al. 1975 (Que)

Trichuris spp.

Site: [intestine]

Hosts: Bos taurus $(3,4,5)$

Canis familiaris $(2,4)$

Ovis aries $(1,4)$

Sus scrofa (2)

Dist.: Alta, NWT, Ont, Sask

Records: 1. Swales et al. 1942 (Ont); 2.

Slocombe 1973a (Ont), 3. 1973b (Ont); 4.

Unruh et al. 1973 (Alta, NWT, Sask); 5.

Moteane et al. 1979 (Sask)

Trichuris spp. (egg)

Site: [intestine]

Hosts: Bos taurus $(1,2)$

Canis familiaris $(3,4,5)$

Dist.: NB, Ont, Que, Sask

Records: 1. McGregor and Kingscote 1957

(Ont); 2. Smith 1970 (NB); 3. Allen and Mills

1971 (Sask); 4. Ghadirian et al. 1976 (Que); 5.

Malloy and Embil 1978 (NB)

Unidentified Trichuridae

Site: [intestine]

Host: Bos taurus

Dist.: Ont

Record: Campbell et al. 1960

\section{SUBCLASS SECERNENTEA}

\section{ORDER ASCARIDIDA}

\section{SUPERFAMILY ASCARIDOIDEA}

FAMILY ASCARIDIDAE

Ascaris lumbricoides Linnaeus, 1758 (larva)

Site: lungs

Hosts: Bos taurus (3)

Sus scrofa $(1,2)$

Dist.: All Provinces, Ont

Records: 1. Swales 1933a (Ont), 2. 1933b (All

Provinces); 3. Allen 1962 (Ont)
Ascaris suum Goeze, 1782

Site: [intestine]

Hosts: Ovis aries (4)

Sus scrofa $(1,2,3)$

Dist.: Nfld, Que, Sask

Records: 1. Smith and Threlfall 1973 (Nfld); 2.

Martin et al. 1974 (Que); 3. Polley and Mostert

1980 (Sask); 4. Sauvageau and Frechette 1980

(Que)

Ascaris spp.

Site: [intestine]

Hosts: Canis familiaris (4)

Ovis aries $(1,2)$

Sus scrofa (3)

Dist.: Canada, Ont, Que

Records: 1. Fallis 1938 (Ont); 2. Swales 1940a

(Canada); 3. Slocombe 1973a (Ont); 4. Seah et al. 1975 (Que)

Parascaris equorum (Goeze, 1782) York and

Site: [intestine] Maplestone, 1926

Host: Equus caballus

Dist.: NB, Nfld, Ont, Que

Records: Swales 1933b (Ont, Que); Smith and Threlfall 1973 (Nfld); Smith 1976a (NB)

Parascaris spp.

Site: [intestine]

Host: Equus caballus

Dist.: Ont

Records: Slocombe 1973a; Slocombe and

McCraw 1973

Toxascaris leonina (Linstow, 1902) Leiper, 1907

Syn.: Toxascaris limbata Railliet and Henry,

1911

Site: intestine

Hosts: Canis familiaris $(1,2,3,4,5,6,7,8,9,10)$ Felis catus $(5,6,10)$

Dist.: Alta, Man, NB, Nfld, NWT, Ont, Que,

Sask

Records: 1. Osler 1877 (Que); 2. Swales 1933b

(Que); 3. Cameron et al. 1940 (Alta, Man, NB,

Nfld, Ont, Que, Sask); 4. Choquette and

Gelinas 1950 (Que); 5. Threlfall 1969 (Nfld); 6.

Smith and Threlfall 1973 (Nfld); 7. Unruh et al.

1973 (Alta, NWT, Sask); 8. Anvik et al. 1974

(Sask); 9. Ghadirian et al. 1976 (Que); 10.

Malloy and Embil 1978 (NB)

Toxascaris spp.

Site: [intestine]

Hosts: Canis familiaris $(1,2)$ Felis catus (2)

Dist.: Ont, Sask

Records: 1. Allen and Mills 1971 (Sask); 2.

Slocombe 1973a (Ont) 
Toxocara canis (Werner, 1782) Stiles, 1905

Site: intestine

Host: Canis familiaris

Dist.: Alta, Man, NB, Nfld, NS, NWT, Ont, Que, Sask

Records: Cameron et al. 1940 (Alta, Man, Nfld, NWT, Ont, Que, Sask); Choquette and Gelinas 1950 (Que); Webster 1958 (Que); Threlfall 1969 (Nfld); Malloy and Embil 1973 (NS); Smith and Threlfall 1973 (Nfld); Unruh et al. 1973 (Alta, NWT, Sask); Anvik et al. 1974 (Sask); Seah et al. 1975 (Que); Ghadirian et al. 1976 (Que); Malloy and Embil 1978 (NB)

Toxocara cati (Schrank, 1788) Brumpt, 1927

Syn.: Belascaris mystax Zeder, 1800

Syn.: Toxocara mystax (Zeder, 1800)

Site: [intestine]

Host: Felis catus

Dist.: NB, Nfld, NS, Que

Records: Swales 1933b (Que); Threlfall 1969 (Nfld); Malloy and Embil 1973(NS); Smith and Threlfall 1973 (Nfld); Malloy and Embil 1978 (NB)

Toxocara spp.

Site: [intestine]

Hosts: Canis familiaris $(1,2)$ Felis catus (2)

Dist.: Ont, Sask

Records: 1. Allen and Mills 1971 (Sask); 2. Slocombe 1973a (Ont)

\section{SUPERFAMILY COSMOCERCOIDEA}

\section{FAMILY ATRACTIDAE}

Probstmayria vivipara (Probstmayr, 1865) Ransom 1907

Site: caecum, colon

Host: Equus caballus

Dist.: NB, Que

Records: Swales 1933a (unspecified), 1933b (Que); Smith 1976a (NB), 1976b exp. (NB), 1979b (NB)

\section{ORDER OXYURIDA}

\section{SUPERFAMILY OXYUROIDEA}

\section{FAMILY OXYURIDAE}

Oxyuris equi (Schrank, 1788) Rudolphi, 1803

Site: [intestine]

Host: Equus caballus

Dist.: NB, Nfld, Ont, Que

Records: Swales 1933b (Ont, Que); Threlfall 1969 (Nfld); Smith 1976a (NB)

Oxyuris spp.

Site: [intestine]

Host: Equus caballus

Dist.: Ont

Records: Slocombe 1973a; Slocombe and

McCraw 1973
Skrjabinema spp.

Site: [intestine]

Host: Ovis aries

Dist.: Sask

Record: Moteane et al. 1979

\section{ORDER RHABDITIDA}

\section{SUPERFAMILY RHABDITOIDEA}

FAMILY RHABDITIDAE

Pelodera strongyloides (Schneider, 1860) Schneider, 1866

Syn.: Rhabditis strongyloides (Schneider, 1860)

Oerley, 1880

Site: unspecified

Host: Canis familiaris

Dist.: Ont

Record: Lord and McGregor 1960

\section{FAMILY STRONGYLOIDIDAE}

Strongyloides papillosus (Wedl, 1856) Ransom, 1911

Site: intestine

Hosts: Bos taurus (7)

Ovis aries $(1,2,3,4,5,6)$

Dist.: Canada, NS, Ont, Que

Records: 1. Griffiths 1937 (Que); 2. Fallis 1938

(Ont); 3. Swales 1940a (Canada), 4. 1940b

(Que); 5. Basir 1950 exp. (Que); 6. Smith and

Archibald 1965 (NS); 7. Frechette and Gibbs 1971 (Que)

Strongyloides stercoralis (Bavay, 1876) Stiles and

Site: unspecified Hassall, 1902

Host: Canis familiaris

Dist.: Que

Records: Choquette and Gelinas 1950; Seah et al. 1975

Strongyloides spp.

Site: unspecified

Hosts: Bos taurus $(3,4)$

Canis familiaris (5)

Equus caballus $(5,6)$

Felis catus (5)

Ovis aries $(1,2,7)$

Sus scrofa (5)

Dist.: Ont, Que, Sask

Records: 1. Swales 1939 (Que); 2. Swales et al. 1942 (Ont); 3. McGregor and Kingscote 1957

(Ont); 4. Slocombe 1973a (Ont), 5. 1973b (Ont);

6. Slocombe and McCraw 1973 (Ont); 7.

Moteane et al. 1979 (Sask)

Unidentified Rhabditoidea

Site: unspecified

Hosts: Bos taurus (1)

Canis familiaris (2)

Dist.: Ont

Felis catus (2)

Records: 1. McGregor and Kingscote 1957; 2.

Slocombe 1973a 
ORDER SPIRURIDA

\section{SUPERFAMILY DRACUNCULOIDEA}

FAMILY DRACUNCULIDAE

Dracunculus insignis (Leidy, 1858) Chandler, 1942

Site: unspecified

Host: Canis familiaris

Dist.: Que

Record: Deroth and Frechette 1975

SUPERFAMILY FILARIOIDEA

FAMILY FILARIIDAE

Parafilaria bovicola Tubangui, 1934

Site: subcutaneous tissue

Host: Bos taurus

Dist.: Alta, Que

Records: Niilo 1968 (Alta); Webster and

Wilkins 1970 (Que)

Remarks: Both records were from imported cattle. Niilo's (1968) record was a presumptive identification.

Stephanofilaria stilesi Chitwood, 1934

Site: subcutaneous

Host: Bos taurus

Dist.: Alta

Record: Maddy 1955; Dies and Pritchard 1985

Remarks: The infection was diagnosed in the

United States in cattle imported from Alberta (Maddy 1955).

\section{FAMILY ONCHOCERCIDAE}

Dipetalonema spp.

Site: unspecified

Host: Canis familiaris

Dist.: Ont

Record: Slocombe 1973a (Ont)

Dirofilaria immitis (Leidy, 1856) Railliet and Henry, 1911

Site: pulmonary artery, right ventricle

Hosts: Canis familiaris $(1,3,4)$

Felis catus (2)

Dist.: Alta, Ont

Records: 1. Fletch 1969 (Ont); 2. Bernard 1970

(Ont), 3. 1971 (Ont); 4. Frimeth and Arai 1983 exp. (Alta)

Remarks: The dog in Bernard's (1971) report was originally diagnosed in the United States.

Dirofilaria spp.

Site: unspecified

Host: Canis familiaris

Dist.: Ont

Record: Slocombe 1973a

Onchocerca cervicalis Railliet and Henry, 1910

Site: unspecified

Host: Equus caballus

Dist.: BC, Que

Records: Marcoux et al. 1977 (Que); Lees et al. 1983 (BC)
Onchocerca gutturosa Neumann, 1910

Site: ligamentum nuchae

Host: Bos taurus

Dist.: Ont, Que

Record: Webster and Dukes 1979

Onchocerca lienalis Stiles, 1892

Site: ligamentum gastrolienale

Host: Bos taurus

Dist.: Ont, Que

Record: Webster and Dukes 1979

Onchocerca spp.

Site: subcutaneous nodules in area of knee joint

Host: Bos taurus

Dist.: Man, Sask

Record: Webster et al. 1977

Setaria equina (Abildgaard, 1789) Railliet and Henry, 1911

Site: unspecified

Host: Equus caballus

Dist.: Ont, Que

Record: Swales 1933b

Setaria labiatopapillosa (Perroncito, 1882) Railliet

Site: unspecified and Henry, 1911

Host: Bos taurus

Dist.: Alta, Man, Ont, Que

Record: Swales 1933b

Setaria spp.

Site: peritoneum

Host: Equus caballus

Dist.: Ont

Record: Slocombe 1973a

SUPERFAMILY HABRONEMATOIDEA

FAMILY HABRONEMATIDAE

Habronema majus (Creplin, 1849) Railliet, 1923

Syn.: Habronema microstoma (Schnider, 1866)

Site: unspecified

Host: Equus caballus

Dist.: Ont

Record: Swales 1933b

Habronema muscae (Carter, 1861) Diesing, 1861

Site: unspecified

Host: Equus caballus

Dist.: Ont

Record: Swales 1933b

Habronema spp.

Site: unspecified

Host: Equus caballus

Dist.: Ont

Record: Slocombe 1973a

SUPERFAMILY PHYSALOPTEROIDEA

FAMILY PHYSALOPTERIDAE

Physaloptera rara Hall and Wigdor, 1918

Site: [intestine]

Host: Canis familiaris

Dist.: Que

Record: Seah et al. 1975 


\section{SUPERFAMILY SPIRUROIDEA}

FAMILY GONGYLONEMATIDAE

Gongylonema pulchrum Molin, 1857

Site: unspecified

Host: Ovis aries

Dist.: Canada, Ont

Records: Swales 1933b (Ont), 1940a (Canada)

\section{FAMILY SPIROCERCIDAE}

Ascarops spp.

Site: stomach

Host: Sus scrofa

Dist.: Ont

Record: Stockdale and Lautenslager 1973

Physocephalus spp.

Site: stomach

Host: Sus scrofa

Dist.: Ont

Record: Stockdale and Lautenslager 1973

Spirocerca spp.

Site: unspecified

Host: Canis familiaris

Dist.: Ont

Record: Slocombe 1973a

SUPERFAMILY THELAZIOIDEA

FAMILY THELAZIIDAE

Thelazia gulosa Railliet and Henry, 1910

Site: unspecified

Host: Bos taurus

Dist.: Ont

Record: Moolenbeek and Surgeoner 1980

Thelazia lacrymalis (Gurlt, 1831) Railliet and Henry, 1910

Site: conjunctiva, nasolacrimal ducts, orbit of eye

Hosts: Bos taurus (2)

Dist.: Ont Equus caballus (1)

Records: 1. Barker 1970; 2. Moolenbeek and

Surgeoner 1980

Thelazia spp.

Site: unspecified

Host: Equus caballus

Dist.: Ont

Record: Slocombe 1973a

\section{ORDER STRONGYLIDA}

\section{SUPERFAMILY ANCYLOSTOMATOIDEA}

\section{FAMILY ANCYLOSTOMATIDAE}

Ancylostomum caninum Ercolani, 1854

Site: [intestine]

Host: Canis familiaris

Dist.: Alta, Ont, Que

Records: Osler 1877 (Que); Swales 1933b (Ont);

Cameron et al. 1940 (Ont, Que); Saunders 1949

(Alta)
Ancylostomum spp.

Site: [intestine]

Hosts: Canis familiaris $(1,2)$

Felis catus (1)

Dist.: Ont, Sask

Records: 1. Slocombe 1973a (Ont); 2. Anvik et al. 1974 (Sask)

Bunostomum phlebotomum (Railliet, 1900) Lane, 1917

Syn.: Monodontus phlebotomus Railliet, 1900

Site: unspecified

Host: Bos taurus

Dist.: Que

Record: Swales 1933b

Bunostomum trigonocephalum (Rudolphi, 1808)

Railliet, 1902

Syn.: Monodontus trigonocephalus Rudolphi, 1808

Site: unspecified

Hosts: Bos taurus (1)

Ovis aries $(1,2,3,4,5,6,7,8)$

Dist.: Canada, Nfld, NS, Ont, Que

Records: 1. Swales 1933b (Ont, Que), 2.

Griffiths 1937 (Que); 3. Swales 1940a (Canada);

4. Fallis 1938 (Ont); 5. Swales and Choquette 1940 (Que); 6. Swales et al. 1942 (Ont); 7. Smith and Archibald 1965 (NS); 8. Smith and

Threlfall 1973 (Nfld)

\section{Bunostomum spp.}

Syn.: Monodontus spp.

Site: unspecified

Hosts: Bos taurus $(2,3)$

Ovis aries $(1,4)$

Dist.: Ont, Que, Sask

Records: 1. Swales 1939 (Que); 2. Frechette and Gibbs 1971 (Que); 3. Slocombe 1973b (Ont); 4.

Moteane et al. 1979 (Sask)

Uncinaria stenocephala (Railliet, 1884) Railliet, 1885

Site: [intestine]

Host: Canis familiaris

Dist.: Alta, BC, Man, Nfld, NWT, Ont, Que

Records: Cameron et al. 1940 (Alta, BC, Man, Nfld, NWT, Ont, Que); Threlfall 1969 (Nfld); Smith and Threlfall 1973 (Nfld); Unruh et al. 1973 (Alta, NWT)

Uncinaria spp.

Site: [intestine]

Host: Canis familiaris

Dist.: Ont, Sask

Records: Slocombe 1973a (Ont); Anvik et al. 1974 (Sask)

Unidentified Ancylostomatidae

Site: unspecified

Host: Canis familiaris

Dist.: Alta, NB, NWT, Que, Sask

Records: Allen and Mills 1971 (Sask); Unruh et al. 1973 (Alta, NWT); Seah et al. 1975 (Que);

Ghadirian et al. 1976 (Que); Malloy and Embil 1978 (NB) 
SUPERFAMILY METASTRONGYLOIDEA

\section{FAMILY ANGIOSTRONGYLIDAE}

Aelurostrongylus spp.

Site: unspecified

Host: Felis catus

Dist.: Ont

Record: Slocombe 1973a

FAMILY CRENOSOMATIDAE

Crenosoma vulpis (Dujardin, 1844) Railliet, 1915

Site: [lungs]

Host: Canis familiaris

Dist.: Nfld

Record: Smith and Threlfall 1973

\section{FAMILY FILAROIDIDAE}

Filaroides milksi Whitlock, 1956

Site: terminal and respiratory bronchioles

Host: Canis familiaris

Dist.: BC

Record: Greenway and Stockdale 1970

Remarks: This was a tentative identification

(Greenway and Stockdale 1970)

Filaroides spp.

Site: unspecified

Host: Canis familiaris

Dist.: Ont

Record: Slocombe 1973a

Oslerus osleri (Cobbold, 1879) Anderson, Chabaud, and Willmott, 1978

Site: bronchi

Host: Canis familiaris

Dist.: Que

Record: Osler 1877

\section{FAMILY METASTRONGYLIDAE}

Metastrongylus pudendotectus Vostokov, 1905

Site: unspecified

Host: Sus scrofa

Dist.: Ont

Record: Swales 1933b

Metastrongylus salmi Gedoelst, 1923

Syn.: Metastrongylus elongatus Salm, 1918

Site: unspecified

Host: Sus scrofa

Dist.: NS, Ont, PEI, Que

Record: Swales 1933b

Metastrongylus spp.

Site: unspecified

Host: Sus scrofa

Dist.: Ont

Record: Slocombe 1973a

FAMILY PROTOSTRONGYLIDAE

Muellerius capillaris (Mueller, 1889) Cameron, 1927

Site: lungs

Host: Ovis aries

Dist.: Canada, Ont

Records: Fallis 1938 (Ont); Swales 1940a

(Canada)
Muellerius spp.

Site: unspecified

Host: Ovis aries

Dist.: Ont

Record: Slocombe 1973a

Parelaphostrongylus tenuis (Dougherty, 1945)

Pryadko and Boev, 1971

Syn.: Pneumostrongylus tenuis Dougherty, 1945

Site: central nervous system

Host: Ovis aries

Dist.: Ont

Record: Anderson and Strelive 1966 exp.

Protostrongylus spp.

Site: unspecified

Host: Ovis aries

Dist.: Ont

Record: Slocombe 1973a

SUPERFAMILY STRONGYLOIDEA

FAMILY CHABERTIIDAE

Chabertia ovina (Fabricius, 1794) Railliet and Henry, 1909

Site: [intestine]

Host: Ovis aries

Dist.: Canada, NS, Ont, Que

Records: Swales 1933b (Ont); Griffiths 1937

(Que); Fallis 1938 (Ont); Swales 1939 (Que);

Swales 1940a (Canada), 1940b (Que); Swales

and Choquette 1940 (Que); Smith and

Archibald 1965 (NS)

Chabertia spp.

Site: [intestine]

Host: Ovis aries

Dist.: Ont, Sask

Records: Swales et al. 1942 (Ont); Slocombe 1973a (Ont); Moteane et al. 1979 (Sask)

Oesophagostomum columbianum (Curtice, 1890)

Site: [intestine]

Stossich, 1899

Host: Ovis arie

Dist.: Canada, NS, Ont, Que

Records: Swales 1933b (NS, Ont, Que); Fallis 1938 (Ont); Swales 1939 (Que), 1940a

(Canada), 1940b (Que); Swales and Choquette

1940 (Que); Swales et al. 1942 (Ont); Smith and Archibald 1965 (NS)

Oesophagostomum dentatum (Rudolphi, 1803)

Site: [intestine]

Molin, 1861

Host: Sus scrofa

Dist.: NB, Ont, Que

Records: Swales 1933b (Ont, Que); Smith 1979a (NB)

Oesophagostomum quadrispinulatum (Marcone, 1901) Alicata, 1935

Site: [intestine]

Host: Sus scrofa

Dist.: NB

Records: Smith 1979a 
Oesophagostomum radiatum (Rudolphi, 1803)

Railliet, 1898

Site: [intestine]

Host: Bos taurus

Dist.: NB, Nfld, Ont, Que

Records: Swales 1933b (Ont, Que); Smith and

Perreault 1972 (NB); Smith and Threlfall 1973 (Nfld)

Oesophagostomum venulosum (Rudolphi, 1809) Railliet and Henry,

Site: [intestine] 1913

Host: Ovis aries

Dist.: Canada, Nfld, NS, Ont, Que

Records: Swales 1933b (Ont, Que), 1940a

(Canada); Smith and Archibald 1965 (NS);

Smith and Threlfall 1973 (Nfld)

Oesophagostomum spp.

Site: intestine

Hosts: Bos taurus $(3,4)$

Ovis aries $(1,4,5)$

Sus scrofa $(2,4,6,7)$

Dist.: NB, Nfld, Ont, Que

Records: 1. Swales 1939 (Que); 2. Davenport and Stockdale 1967 (Ont); 3. Frechette and Gibbs 1971 (Que); 4. Slocombe 1973b (Ont); 5.

Smith and Threlfall 1973 (Nfld); 6. Martin et al. 1974 (Que); 7. Smith 1979a (NB)

\section{FAMILY STRONGYLIDAE}

\section{Cyathostomum catinatum Looss, 1900}

Syn.: Cylicostomum catinatum (Looss, 1900)

Gedoelst, 1924

Syn.: Trichonema catinatum (Looss, 1900)

LeRoux, 1924

Site: [intestine]

Host: Equus caballus

Dist.: Alta, Nfld, Sask

Records: Ransom and Hadwen 1918 (Alta,

Sask); Smith and Threlfall 1973 (Nfld)

\section{Cyathostomum coronatum Looss, 1900}

Syn.: Cylicostomum coronatum (Looss, 1900)

Gedoelst, 1903

Site: [intestine]

Host: Equus caballus

Dist.: Sask

Record: Ransom and Hadwen 1918

Cyathostomum labiatum (Looss, 1902) McIntosh, 1933

Syn.: Cylicostomum labiatum (Looss, 1902)

Gedoelst, 1903

Site: [intestine]

Host: Equus caballus

Dist.: Alta, Sask

Record: Ransom and Hadwen 1918
Cyathostomum labratum Looss, 1900

Syn.: Cylicostomum labratum (Looss, 1900)

Gedoelst, 1903

Site: [intestine]

Host: Equus caballus

Dist.: Alta, Sask

Record: Ransom and Hadwen 1918

Cylicocyclus elongatus (Looss, 1900) Chaves, 1930

Syn.: Cylicostomum elongatum (Looss, 1900)

Gedoelst, 1903

Site: [intestine]

Host: Equus caballus

Dist.: Nfld, Sask

Records: Ransom and Hadwen 1918 (Sask);

Smith and Threlfall 1973 (Nfld)

Cylicocyclus insigne (Boulenger, 1917) Chaves, 1930

Syn.: Cylicostomum insigne (Boulenger, 1917)

Ransom and Hadwen, 1918

Site: [intestine]

Host: Equus caballus

Dist.: Alta

Record: Ransom and Hadwen 1918

Cylicocyclus nassatus (Looss, 1900) Chaves, 1930

Syn.: Cylicostomum nassatum (Looss, 1900)

Gedoelst, 1903

Site: [intestine]

Host: Equus caballus

Dist.: Alta, Nfld, Sask

Records: Ransom and Hadwen 1918 (Alta,

Sask); Smith and Threlfall 1973 (Nfld)

Cylicocyclus radiatus (Looss, 1900) Chaves, 1930

Syn.: Cylicostomum radiatum (Looss, 1900)

Gedoelst, 1903

Site: [intestine]

Host: Equus caballus

Dist.: Alta, Sask

Record: Ransom and Hadwen 1918

Cylicodontophorus bicornatus (Looss, 1900) Cram, 1924

Syn.: Cylicostomum bicornatum (Looss, 1900)

Gedoelst, 1903

Site: [intestine]

Host: Equus caballus

Dist.: Alta, Sask

Record: Ransom and Hadwen 1918

Cylicostephanus calicatus (Looss, 1900) Cram, 1924

Syn.: Cylicostomum calicatum (Looss, 1900)

Gedoelst, 1903

Site: [intestine]

Host: Equus caballus

Dist.: Alta, Sask

Record: Ransom and Hadwen 1918 
Cylicostephanus goldi (Boulenger, 1917) Lichtenfels, 1975

Syn.: Cylicostomum goldi (Boulenger, 1917)

Ransom and Hadwen, 1918

Site: [intestine]

Host: Equus caballus

Dist.: Alta, Sask

Record: Ransom and Hadwen 1918

Cylicostephanus poculatus (Looss, 1900) Cram, 1924

Syn.: Cylicostomum poculatum Looss, 1900

Syn.: Petrovinema poculatum (Looss, 1900)

Ershov, 1943

Site: [intestine]

Host: Equus caballus

Dist.: Alta, Nfld, Sask

Records: Ransom and Hadwen 1918 (Alta,

Sask); Smith and Threlfall 1973 (Nfld)

Gyalocephalus capitatus Looss, 1900

Site: unspecified

Host: Equus caballus

Dist.: Alta, NB, Ont, Sask

Records: Ransom and Hadwen 1918 (Alta,

Sask); Swales 1933b (Ont); Smith 1976a (NB)

Gyalocephalus spp.

Site: unspecified

Host: Equus caballus

Dist.: NB

Record: Smith 1976a

Oesophagodontus robustus (Giles, 1892) Railliet and Henry, 1902

Site: unspecified

Host: Equus caballus

Dist.: Alta, Nfld, Ont, Que, Sask

Records: Ransom and Hadwen 1918 (Alta,

Sask); Swales 1933b (Ont, Que); Threlfall 1969

(Nfld)

Oesophagodontus spp.

Site: unspecified

Host: Equus caballus

Dist.: NB

Record: Smith 1976a

Poteriostomum spp.

Site: unspecified

Host: Equus caballus

Dist.: NB

Record: Smith 1976a (NB)

Strongylus edentatus (Looss, 1900) Railliet and Henry, 1909

Syn.: Alfortia edentatus (Looss, 1900) Skrjabin, 1933

Site: aorta

Host: Equus caballus

Dist.: Alta, NB, Nfld, Ont, Que, Sask

Records: Ransom and Hadwen 1918 (Alta);

Swales 1933a (Sask), 1933b (Que); Slocombe

1973a (Ont); Slocombe and McCraw 1973

(Ont); Smith and Threlfall 1973 (Nfld); Smith

1976a (NB), 1976b exp. (NB)
Strongylus equinus Mueller, 1780

Site: unspecified

Host: Equus caballus

Dist.: Alta, NB, Ont, Que, Sask

Records: Ransom and Hadwen 1918 (Alta);

Swales 1933a (Sask), 1933b (Ont, Que);

Slocombe and McCraw 1973 (Ont); Smith

1976a (NB), 1976b exp. (NB)

Strongylus vulgaris (Looss, 1900) Railliet and Henry, 1909

Syn.: Delafondia vulgaris (Looss, 1900)

Skrjabin, 1933

Site: cranial mesenteric arteries, central nervous system

Host: Equus caballus

Dist:: Alta, NB, Nfld, Ont, Que, Sask

Records: Ransom and Hadwen 1918 (Alta, Sask); Swales 1933a (Sask); Little 1972 (Ont);

Slocombe 1973a (Ont); Smith and Threlfall

1973 (Nfld); Smith 1976a (NB), 1976b exp.

(NB)

Remarks: Little (1972) presumed the larva he recovered from the central nervous system of the horse was $S$. vulgaris.

Strongylus spp.

Syn.: Alfortia spp.

Site: unspecified

Host: Equus caballus

Dist.: NB, Ont

Records: Slocombe 1973a (Ont); Smith 1976a (NB)

Triodontophorus brevicauda Boulenger, 1916

Site: unspecified

Host: Equus caballus

Dist.: Alta, Que, Sask

Records: Ransom and Hadwen 1918 (Alta,

Sask); Swales 1933b (Que)

Triodontophorus minor (Looss, 1900) Looss, 1902

Site: unspecified

Host: Equus caballus

Dist.: Nfld

Record: Smith and Threlfall 1973

Triodontophorus serratus (Looss, 1900) Looss, 1902

Syn.: Triodontophorus intermedius Sweet, 1909

Site: unspecified

Host: Equus caballus

Dist.: Alta, Nfld, Ont, Que, Sask

Records: Ransom and Hadwen 1918 (Alta,

Sask); Swales 1933a (Sask), 1933b (Ont, Que);

Smith and Threlfall 1973 (Nfld)

Triodontophorus tenuicollis Boulenger, 1916

Site: unspecified

Host: Equus caballus

Dist.: Alta, Sask

Record: Ransom and Hadwen 1918 
Triodontophorus spp.

Site: unspecified

Host: Equus caballus

Dist.: NB, Ont

Records: Slocombe 1973a (Ont); Smith 1976a

(NB), 1976b exp. (NB)

Unidentified Strongylidae

Site: unspecified

Host: Equus caballus

Dist.: NB, Ont, Que

Records: Swales 1933b (Ont, Que); Slocombe and McCraw 1973 (Ont); Smith 1976a (NB),

1976b exp. (NB)

\section{FAMILY SYNGAMIDAE}

Cyathostoma spp.

Site: [intestine]

Host: Equus caballus

Dist.: Nfld

Record: Threlfall 1969

Stephanurus dentatus Diesing, 1839

Site: kidney

Host: Sus scrofa

Dist.: Man, NS

Records: Swales 1933b (Man); Smith and

Hawkes 1978 (NS)

\section{SUPERFAMILY TRICHOSTRONGYLOIDEA}

\section{FAMILY DICTYOCAULIDAE}

Dictyocaulus arnfieldi (Cobbold, 1884) Railliet and

Site: [lungs] Henry, 1907

Host: Equus caballus

Dist.: Ont

Records: Swales 1933b

Dictyocaulus filaria (Rudolphi, 1809) Railliet and

Site: [lungs]

$$
\text { Henry, } 1907
$$

Host: Ovis aries

Dist.: Alta, Canada, Nfld, Ont, PEI, Que

Records: Swales 1933b (Ont, PEI, Que); Fallis 1938 (Ont); Swales 1940a (Canada);

O’Donoghue 1958 (Alta); Smith and Threlfall 1973 (Nfld)

Dictyocaulus viviparus (Bloch, 1782) Railliet and

Site: lungs

$$
\text { Henry, } 1907
$$

Host: Bos taurus

Dist.: Alta, Nfld, Ont, Que

Records: Swales 1933b (Ont, Que); Campbell and Wetherill 1957 (Ont); Graesser 1957 (Alta); O’Donoghue 1958 (Alta); Smith and Threlfall 1973 (Nfld)
Dictyocaulus spp.

Site: [lungs]

Hosts: Bos taurus $(1,2)$

Dist.: Ont

Equus caballus (2)

Ovis aries (2)

Records: 1. McGregor and Kingscote 1957; 2.

Slocombe 1973a

\section{FAMILY HELIGMOSOMIDAE}

Citellinema bifurcatum Hall, 1916

Syn.: Warrenius bifurcatus (Hall, 1916) Sleggs, 1925

Site: unspecified

Host: Equus caballus

Dist.: Sask

Record: Swales 1933a

\section{FAMILY MOLINEIDAE}

Nematodirus abnormalis May, 1920

Site: unspecified

Host: Ovis aries

Dist.: Nfld

Record: Smith and Threlfall 1973

Nematodirus filicollis Rudolphi, 1802

Site: [intestine]

Host: Ovis aries

Dist.: Canada, NS, Ont, Que

Records: Griffiths 1937 (Que); Fallis 1938

(Ont); Swales 1940a (Canada), 1940b (Que);

Swales and Choquette 1940 (Que); Smith and

Archibald 1965 (NS)

Nematodirus helvetianus May, 1920

Site: [intestine]

Hosts: Bos taurus

Ovis aries

Dist.: NB, Ont

Records: 1. Swales 1933b (Ont); 2. Campbell et al. 1960 (Ont); 3. Smith and Archibald 1968

(NB); 4. Smith 1970 (NB)

Nematodirus spathiger (Railliet, 1896) Railliet and Henry, 1909

Site: intestine

Hosts: Equus caballus (6) Ovis aries $(1,2,3,4,5,7)$

Dist.: Canada, Nfld, NS, Ont, Que

Records: 1. Swales 1933b (Ont); 2. Fallis 1938

(Ont); 3. Swales 1940a (Canada); 4. Swales and Choquette 1940 (Que); 5. Smith and Archibald 1965 (NS); 6. Webster and MacKay 1969

(Que); 7. Smith and Threlfall 1973 (Nfld) 
Nematodirus spp.

Site: unspecified

Hosts: Bos taurus $(3,4,5)$

Ovis aries $(1,2,6)$

Dist.: Ont, Que, Sask

Records: 1. Swales 1939 (Que); 2. Swales et al.

1942 (Ont); 3. McGregor and Kingscote 1957

(Ont); 4. Frechette and Gibbs 1971 (Que); 5.

Slocombe 1973b (Ont); 6. Moteane et al. 1979

(Sask)

Ollulanus tricuspis Leuckart, 1865

Site: stomach

Hosts: Felis catus $(1,3,4)$

Sus scrofa (2)

Dist.: Alta, Ont, Que

Records: 1. Cameron 1940b (Que); 2. Stockdale and Lautenslager 1973 (Ont); 3. Caldwell 1984

(Ont); 4. Kennedy et al. 1984 (Alta)

\section{FAMILY TRICHOSTRONGYLIDAE}

Cooperia curticei (Railliet, 1893) Ransom, 1907

Site: [intestine]

Host: Ovis aries

Dist.: Canada, NS, Ont, Que

Records: Swales 1933b (Ont); Fallis 1938 (Ont);

Swales 1940a (Canada), 1940b (Que); Swales and Choquette 1940 (Que); Smith and

Archibald 1965 (NS)

Cooperia oncophora (Railliet, 1898) Ransom, 1907

Site: [intestine]

Hosts: Bos taurus $(5,6,7)$

Ovis aries $(1,2,3,4)$

Dist.: Canada, NB, NS, Ont

Records: 1. Swales 1933b (Ont); 2. Fallis 1938

(Ont); 3. Swales 1940a (Canada); 4. Smith and

Archibald 1965 (NS), 5. 1968 (NB); 6. Smith

1970 (NB); 7. Smith and Threlfall 1973 (Nfld)

Cooperia punctata (von Linstow, 1907) Ransom, 1907

Site: [intestine]

Host: Bos taurus

Dist.: Ont

Record: Swales 1933b

Cooperia spp.

Site: [intestine]

Hosts: Bos taurus $(3,4)$

Ovis aries $(1,2,5)$

Dist.: Ont, Que, Sask

Records: 1. Swales 1939 (Que); 2. Swales et al. 1942 (Ont); 3. Frechette and Gibbs 1971 (Que); 4. Slocombe 1973a (Ont); 5. Moteane et al. 1979 (Sask)
Haemonchus contortus (Rudolphi, 1803) Cobb, 1898

Site: intestine

Hosts: Bos taurus $(1,7,8)$

Ovis aries $(1,2,3,4,5,6,9,10,11,12)$

Dist.: Canada, NS, Ont, Prairie Provinces, Que

Records: 1. Swales 1933b (Ont, Que); 2.

Griffiths 1937 exp. (Que); 3. Fallis 1938 (Ont);

4. Swales 1940a (Canada), 5. 1940b (Que); 6.

Swales and Choquette 1940 (Que); 7.

Choquette 1945 (Ont, Prairie Provinces, Que); 8. Johnston and MacPherson 1956 (Ont); 9.

Gibbs 1964 (Que); 10. Smith and Archibald 1965 (NS); 11. Blitz and Gibbs 1971 (Que); 12. Slocombe 1973c (Ont)

Haemonchus spp.

Site: [intestine]

Hosts: Bos taurus $(4,5)$

Ovis aries $(1,2,3,4,6)$

Dist.: Ont, Que, Sask

Records: 1. Swales 1933b (Ont), 2. 1939 (Que); 3. Swales et al. 1942 (Ont); 4. Slocombe 1973b (Ont), 5. 1974 (Ont); 6. Moteane et al. 1979 (Sask)

Hyostrongylus rubidus (Hassall and Stiles, 1892) Hall, 1921

Site: stomach

Host: Sus scrofa

Dist.: Ont, Que

Records: 1. Swales 1933b (Ont); 2. Davenport and Stockdale 1967 (Ont); 3. Stockdale,

Ashton et al. 1973 (Ont); 4. Stockdale and Lautenslager 1973 (Ont); 5. Martin et al. 1974 (Que)

Hyostrongylus spp.

Site: [stomach]

Host: Sus scrofa

Dist.: Ont

Record: Slocombe 1973a

Ostertagia circumcincta (Stadelmann, 1894) Ransom, 1907

Site: [intestine]

Hosts: Bos taurus (11)

Ovis aries $(1,2,3,4,5,6,7,8,9,10)$

Dist.: Canada, Nfld, NS, Ont, Que

Records: 1. Swales 1933b (Ont); 2. Griffiths 1937 (Ont); 3. Fallis 1938 (Ont); 4. Swales 1939 (Que), 5. 1940a (Canada), 6. 1940b (Que); 7

Swales and Choquette 1940 (Que); 8. Swales et al. 1942 (Ont); 9. Smith and Archibald 1965 (NS); 10. Swales and Threlfall 1973 (Nfld); 11. Slocombe 1974 (Ont)

Ostertagia lyrata Sjberg, 1926

Site: [intestine]

Host: Bos taurus

Dist.: Ont

Record: Slocombe 1974 
Ostertagia ostertagi (Stiles, 1892) Ransom, 1907

Site: [intestine]

Host: Bos taurus

Dist.: NB, Ont, Que

Records: Swales 1933b (Que); Johnston and

MacPherson 1956 (Ont); Smith and Archibald

1968 (NB); Smith 1970 (NB); Smith and

Perreault 1972 (NB); Slocombe 1974 (Ont)

Ostertagia trifurcata Ransom, 1907

Site: [intestine]

Hosts: Bos taurus $(2,3)$ Ovis aries (1)

Dist.: Alta, NS, Ont

Records: 1. Smith and Archibald 1965 (NS); 2.

Slocombe 1970 (Alta), 3. 1974 (Ont)

Remarks: In Slocombe (1970), the ox originated in Alberta and was slaughtered in Ontario.

Ostertagia spp.

Site: [intestine]

Hosts: Bos taurus $(3,4)$

Ovis aries $(1,2,4,5)$

Dist.: Ont, Que, Sask

Records: 1. Swales 1940b exp. (Que); 2. Gibbs 1964 (Que); 3. Frechette and Gibbs 1971 (Que);

4. Slocombe 1973b (Ont); 5. Moteane et al. 1979 (Sask)

Teladorsagia davtiani Andreeva and Satubaldin, 1954

Site: [intestine]

Host: Ovis aries

Dist.: NS

Record: Smith and Archibald 1965

Trichostrongylus axei (Cobbold, 1879) Railliet and Henry, 1909

Syn.: Trichostrongylus extenuatus (Railliet, 1898) Ransom, 1907

Site: [intestine]

Hosts: Bos taurus $(10,11,12,13)$ Ovis aries $(1,2,3,4,5,6,7,8,9,12)$

Dist.: Canada, NB, NS, Ont, Que

Records: 1. Swales 1933b (Ont), 2. 1939 (Que);

3. Fallis 1938 (Ont); 4. Swales 1940a (Canada),

5. 1940b (Que); 6. Swales and Choquette 1940

(Que); 7. Swales et al. 1942 (Ont); 8. Gibbs 1964

(Que); 9. Smith and Archibald 1965 (NS); 10.

Smith 1970 (NB); 11. Frechette and Gibbs 1971

(Que); 12. Slocombe 1973a (Ont), 13. 1974

(Ont)

Trichostrongylus colubriformis (Giles, 1892)

Site: [intestine]

Ransom, 1911

Host: Ovis aries

Dist.: Canada, NS, Ont, Que

Records: Swales 1933a (Ont); Griffiths 1937

(Que); Fallis 1938 (Ont); Swales 1939 (Que),

1940a (Canada), 1940b (Que); Swales and

Choquette 1940 (Que); Smith and Archibald 1965 (NS)
Trichostrongylus vitrinus Looss, 1905

Site: [intestine]

Host: Ovis aries

Dist.: Canada, NS, Ont, Que

Records: Swales 1933a (Ont); Fallis 1938 (Ont);

Swales 1939 (Que), 1940a (Canada); Swales

and Choquette 1940 (Que); Smith and

Archibald 1965 (NS)

Trichostrongylus spp.

Site: [intestine]

Hosts: Bos taurus (4)

Ovis aries $(1,2,3,4,5)$

Dist.: Ont, Que, Sask

Records: 1. Griffiths 1937 (Que); 2. Swales 1939

(Que); 3. Swales et al. 1942 (Ont); 4. Slocombe

1973b (Ont); 5. Moteane et al. 1979 (Sask)

Unidentified Trichostrongyloidea (egg)

Site: [intestine]

Host: Bos taurus

Dist.: Ont

Record: McGregor and Kingscote 1957

Unidentified Nematoda

Site: unspecified

Hosts: Bos taurus (2)

Equus caballus (1)

Dist.: Nfld, Que

Records: 1. Threlfall 1969 (Nfld); 2. Frechette and Gibbs 1971 (Que)

\section{PHYLUM ACANTHOCEPHALA}

CLASS ARCHIACANTHOCEPHALA

ORDER OLIGACANTHORHYNCHIDA

\section{FAMILY OLIGACANTHORHYNCHIDAE}

Macracanthorhynchus hirudinaceus (Pallas, 1781)

Site: [intestine]

Host: Sus scrofa

Dist.: Ont

Record: Swales 1933b

CLASS PALAEACANTHOCEPHALA

\section{ORDER POLYMORPHIDA}

\section{FAMILY POLYMORPHIDAE}

Corynosoma semerme (Forssell, 1904)

Site: [intestine]

Host: Canis familiaris

Dist.: NWT

Record: Cameron et al. 1940 


\section{PHYLUM CHELICERATA}

CLASS ARACHNIDA

SUBCLASS ACARI

ORDER ACARIFORMES

SUBORDER ACARIDIDA

\section{FAMILY PSOROPTIDAE}

Chorioptes bovis (Hering, 1845) Gervais and Van

Site: unspecified

Beneden, 1859

Hosts: Bos taurus

Dist.: Ont

Equus caballus

Record: Slocombe 1973a

Remarks: Slocombe (1973a) recorded the mite as Chorioptes spp.

Otodectes cynotis (Hering, 1838) Canestrini, 1894

Site: ear

Hosts: Canis familiaris (2)

Felis catus $(1,2,3)$

Dist.: BC, NB, Que

Records: 1. Spencer 1940 (BC); 2. Sweatman 1958 exp. (Que); 3. Malloy and Embil 1978 (NB)

Otodectes cynotis (Hering) var. canis Sewell

Host: Canis familiaris

Dist.: BC

Record: Spencer 1939

Otodectes cynotis (Hering) var. felis Huber

Host: Felis catus

Dist: BC

Record: Spencer 1939

Psoroptes spp.

Site: unspecified

Host: Equus caballus

Dist.: Ont

Record: Slocombe 1973a

\section{FAMILY SARCOPTIDAE}

Notoedres spp.

Site: unspecified

Host: Canis familiaris

Dist.: Ont

Record: Slocombe 1973a

Sarcoptes scabiei (Linnaeus, 1758)

Site: unspecified

Hosts: Bos taurus (2)

Canis familiaris (2)

Felis catus (1)

Dist.: Ont

Sus scrofa (2)

Record: 1. Tyrrell 1883 (unspecified); 2.

Slocombe 1973a (Ont)

\section{SUBORDER ACTINEDIDA}

\section{FAMILY CHEYLETIDAE}

Cheyletiella spp.

Site: unspecified

Host: Canis familiaris

Dist.: Ont

Record: Slocombe 1973a

\section{FAMILY DEMODICIDAE}

Demodex bovis Stiles, 1892

Site: [skin]

Host: Bos taurus

Dist.: Ont

Record: Smith 1961

Demodex phylloides Croker

Host: Sus scrofa

Dist.: Ont

Records: Jarvis 1910; MacNay 1952

Remarks: MacNay (1952) felt his specimens were probably $D$. phylloides.

Demodex spp.

Site: unspecified

Hosts: Bos taurus

Dist.: Ont

Canis familiaris

Record: Slocombe 1973a

\section{FAMILY TROMBICULIDAE}

Unidentified Trombiculidae

Site: unspecified

Host: Canis familiaris

Dist.: Ont

Record: Slocombe 1973a

ORDER PARASITIFORMES

SUBORDER GAMASIDA

FAMILY DERMANYSSIDAE

Pneumonyssus spp.

Site: Unspecified

Host: Canis familiaris

Dist.: Ont

Record: Slocombe 1973a

\section{SUBORDER IXODIDA}

FAMILY ARGASIDAE

Otobius lagophilus Cooley and Kohls, 1940

Site: unspecified

Host: Felis catus

Dist.: Alta

Record: Brown 1944b 
Otobius megnini (Dugs, 1883) Banks, 1912

Site: unspecified

Hosts: Bos taurus $(2,3)$

Felis catus $(1,3)$

Ovis aries (2)

Dist.: BC

Record: 1. Gregson 1953; 2. MacNay 1957; 3.

Rich 1957

Otobius spp.

Site: unspecified

Host: Canis familiaris

Dist.: Ont

Record: Slocombe 1973a

\section{FAMILY IXODIDAE}

Dermacentor albipictus (Packard, 1869) Banks, 1908

Syn.: Dermacentor erraticus var. albipictus

(Packard)

Site: skin

Hosts: Bos taurus $(1,2,4,5,9)$

Canis familiaris (8)

Equus caballus $(1,2,3,4,5,6,7,8)$

Dist.: Alta, BC, NB, NS, Sask

Records: 1. Hewitt 1915 (BC, NB); 2. Cameron and Fulton 1927 (Sask); 3. Hearle 1933 (BC), 4. 1938 (BC); 5. Bequaert 1945 (NB, NS); 6.

Brown and Kohls 1950 (Alta); 7. MacNay 1952 (BC), 8. 1957 (Sask); 9. Wilkinson and Lawson 1965 (unspecified)

Dermacentor andersoni Stiles, 1905

Syn.: Dermacentor venustus Banks, 1908

Site: skin

Hosts: Bos taurus $(3,7,10,11,12,14,15,17,18,19)$

Canis familiaris $(6,13)$

Equus caballus $(2,6,7,13)$

Felis catus (13)

Ovis aries $(1,4,5,6,7,8,9,11,13)$

Sus scrofa (13)

unspecified (12)

Dist.: Alta, BC, Sask

Records: 1. Hadwen 1913 (BC); 2. Hewitt 1915

(Alta, BC); 3. Hearle 1933 (BC); 4. Gregson

1935 (BC), 5. 1937 exp. (BC); 6. Hearle 1938

(BC); 7. Holland 1940 (Alta); 8. Gregson 1947

exp. (BC), 9. 1952 exp. (BC); 10. MacNay 1952

(BC, Sask); 11. Weintraub 1952 exp. (BC); 12.

MacNay 1957 (BC); 13. Gregson 1958a exp.

(BC); 14. Gregson 1958c (BC); 15. Wilkinson and Lawson 1965 (Alta, BC); 16. Wilkinson 1968 exp. (BC), 17. 1972 exp. (Alta, BC, Sask), 18. 1981 (Alta, BC), 19. 1982 exp. (BC)

Dermacentor variabilis (Say, 1821) Banks, 1908

Site: unspecified

Host: Canis familiaris $(1,2,3)$ collected by flagging (4)

Dist.: Man, NS, Ont

Records: 1. Bequaert 1945 (Ont); 2. MacNay 1952 (Man); 3. Judd 1953 (Ont); 4. Hall and McKiel 1961 (NS)
Dermacentor spp.

Site: unspecified

Hosts: Felis catus

Dist.: Ont

Equus caballus

Record: Slocombe 1973a

Haemaphysalis cinnabarina Koch

Host: Bos taurus

Dist.: Man

Record: Hewitt 1915

Haemaphysalis leporis-palustris (Packard)

Host: Felis catus

Dist.: BC

Record: Hearle 1938

Ixodes cookei Packard, 1869

Syn.: Ixodes hexagonus var. cookei Packard

Hosts: Bos taurus (3)

Canis familiaris $(1,2,3,4)$

Felis catus $(3,5)$

Dist.: BC, NB, Ont, Que

Records: 1. Hewitt 1915 (Ont); 2. Hearle 1938

(BC); 3. Bequaert 1945 (NB, Ont, Que); 4.

Cooley and Kohls 1945 (Ont); 5. Judd 1954

(Ont)

Ixodes ricinus Linnaeus

Host: Canis familiaris

Dist.: BC

Records: Hewitt 1915; Hearle 1938

Ixodes ricinus var. scapularis Say

Host: Canis familiaris

Dist.: BC

Record: Hearle 1938

Ixodes spp.

Site: unspecified

Hosts: Canis familiaris

Dist.: Ont

Felis catus

Record: Slocombe 1973a

Rhipicephalus sanguineus (Latreille, 1806) Koch, 1844

Site: unspecified

Host: Canis familiaris

Dist.: BC, NS, Ont

Record: Bequaert 1945 (NS, Ont); MacNay 1957 (Ont); Spencer 1960 (BC); Wright 1979

(NS)

Rhipicephalus spp.

Site: unspecified

Host: Canis familiaris

Dist.: Ont

Record: Slocombe 1973a 


\section{PHYLUM UNIRAMIA}

\section{CLASS INSECTA}

ORDER DIPTERA

\section{FAMILY CALLIPHORIDAE}

Calliphora spp.

Host: unspecified

Dist.: BC

Record: MacNay 1952

Lucilia illustris Meigen, 1826

Site: unspecified

Host: Ovis aries

Dist.: Ont

Record: Teskey 1960

Lucilia sericata (Meigen, 1826)

Syn.: Phaenicia sericata (Meigen, 1826)

Host: Ovis aries

Dist.: Nfld

Record: MacNay 1957

Phormia regina Meigen, 1826

Site: unspecified

Hosts: Bos taurus (1)

Ovis aries (2)

Dist.: BC, Ont

Records 1. Hearle 1933 (BC); 2. Teskey 1960 (Ont)

Phormia terranovae Robineau-Desvoidy, 1830

Syn.: Protophormia terranovae (Robineau-

Desvoidy, 1830) Townsend, 1908

Site: unspecified

Host: Ovis aries

Dist.: Ont

Record: Teskey 1960

Wohlfahrtia opaca (Coquillet)

Host: unspecified

Dist.: BC

Record: MacNay 1952

Wohlfahrtia vigil (Walker, 1949)

Host: unspecified

Dist.: BC

Record: MacNay 1952

Unidentified Calliphoridae

Site: unspecified

Host: Felis catus

Dist.: Ont

Record: Slocombe 1973a

\section{FAMILY CUTEREBRIDAE}

Cuterebra spp.

Site: unspecified

Hosts: Canis familiaris (2)

Dist.: Ont

Felis catus $(1,2)$

Records: 1. Scholten et al. 1962; Slocombe 1973a
FAMILY GASTEROPHILIDAE

Gasterophilus intestinalis (De Geer, 1776) Leach, 1817

Site: mouth

Host: Equus caballus $(2,3,4)$ unspecified (1)

Dist.: Alta, Ont, Sask

Records: 1. MacNay 1952 (Sask); 2. Nelson 1952 (Alta); 3. Teskey 1960 (Ont); 4. Slocombe 1973a (Ont)

Gasterophilus nasalis (Linnaeus, 1758) Leach, 1817

Site: mouth

Host: Equus caballus

Dist.: Alta, Ont

Records: Nelson 1952 (Alta); Teskey 1960

(Ont); Slocombe 1973a (Ont)

Gasterophilus spp.

Host: Equus caballus

Dist.: Man

Record: MacNay 1952

\section{FAMILY HIPPOBOSCIDAE}

Melophagus ovinus (Linnaeus, 1758) Latreille, 1802 Site: skin

Host: Ovis aries

Dist.: BC, Ont, Sask

Records: Spencer 1938 (BC), 1939 (BC);

MacNay 1952 (BC); Teskey 1960 (Ont);

Slocombe 1973a (Ont); Moteane et al. 1979 (Sask)

\section{FAMILY OESTRIDAE}

Hypoderma bovis (Linnaeus, 1758) Latreille, 1818

Site: subcutaneous tissues

Host: Bos taurus

Dist.: Alta, BC, Ont

Records: Gregson 1958b (Alta, BC); Teskey 1960 (Ont)

Hypoderma lineatum (Villers, 1789) Latreille, 1818

Site: subcutaneous tissues

Host: Bos taurus

Dist.: Alta, BC

Records: Gregson 1958b (Alta, BC); Nelson and Weintraub 1972 exp. (Alta)

Hypoderma spp.

Site: oesophagus, subcutaneous tissues

Hosts: Bos taurus $(2,3)$

Equus caballus (2)

unspecified (1)

Dist.: Alta, BC, Man, Ont, Sask

Records: 1. MacNay 1957 (Alta, BC, Man, Ont); 2. Slocombe 1973a (Ont); 3. Blakley 1979 (Sask) 
Oestrus ovis Linnaeus, 1761

Site: unspecified

Host: Ovis aries

Dist.: Ont, Western Canada

Records: Fallis 1940 (Ont, Western Canada);

Teskey 1960 (Ont); Slocombe 1973a (Ont)

Unidentified Diptera

Site: lung

Host: Ovis aries

Dist.: BC

Record: MacNay 1952

Remarks: One rat-tailed maggot found in the lung of a sheep (MacNay 1952).

\section{ORDER MALLOPHAGA}

\section{SUBORDER ISCHNOCERA}

Damalinia bovis (Linnaeus, 1758) Mjoberg, 1910

Syn.: Bovicola bovis

Site: unspecified

Host: Bos taurus

Dist.: BC, Man, NS, Ont, Que

Record: Thompson 1934 (NS, Que);

Whitehead 1934 (Que); Spencer 1939 (BC);

MacNay 1952 (Man); Hopkins 1960 (BC);

Teskey 1960 (Ont)

Damalinia caprae (Gurlt, 1843)

Syn.: Bovicola caprae (Gurlt, 1843)

Host: Capra hircus

Dist.: BC

Record: Spencer 1939

Damalinia equi (Denny, 1842)

Host: Equus cabalus

Dist.: BC

Record: Hopkins 1960

Damalinia limbata (Gervais, 1844) Mjoberg, 1910

Site: unspecified

Host: Capra hircus

Dist.: Ont

Record: Teskey 1960

Damalinia spp.

Site: unspecified

Hosts: Bos taurus

Dist.: Ont

Equus caballus

Record: Slocombe 1973a

Felicola subrostratus (Nitzsch, 1838)

Host: Felis catus

Dist.: BC, Que

Records: Whitehead 1934 (Que); Hopkins 1960

(BC)

Trichodectes canis (de Geer, 1778)

Host: Canis familiaris

Dist.: BC

Records: Spencer 1939; Hopkins 1960
Trichodectes equi (Linnaeus)

Host: Equus caballus

Dist.: BC

Record: Spencer 1939

Trichodectes spp.

Site: unspecified

Host: Canis familiaris

Dist.: Ont

Record: Slocombe 1973a

ORDER SIPHONAPTERA

FAMILY CERATOPHYLLIDAE

Monopsyllus vison (Baker, 1904)

Host: Felis catus

Dist.: Sask

Record: Holland 1984

FAMILY HYSTRICHOPSYLLIDAE

Tamiophila grandis (Rothschild, 1902)

Host: Canis familiaris

Dist.: NB

Record: Brown 1968

\section{FAMILY PULICIDAE}

Ctenocephalides canis (Curtis, 1826)

Hosts: Canis familiaris $(1,2,3,4,5,6)$

Felis catus $(3,4)$

Dist.: Alta, BC, NB, Ont

Records: 1. Harvey 1907 (BC); 2. Spencer 1936

(BC), 3. 1939 (BC); 4. Brown 1944a (Alta); 5.

Brown 1955 (NB); 6. Holland 1984 (BC, Ont)

Ctenocephalides felis (Bouche, 1835)

Hosts: Canis familiarus $(2,3)$

Felis catus $(1,2,3)$

Dist.: BC, NS

Records: 1. Spencer 1936 (BC), 2. 1939 (BC); 3.

Wright 1979 (NS)

Ctenocephalides felis felis (Bouch, 1835)

Site: unspecified

Hosts: Canis familiaris $(2,3,4)$

Felis catus $(1,2,3,4)$

Dist.: Alta, BC, Man, Nfld, NB, NS, Ont, Que, Sask

Record: 1. Brown 1944a (Alta); 2. Brown 1955

(NB, NS); 3. Buckner 1964 (Man); 4. Holland

1984 (BC, Man, Nfld, NS, Ont, Que, Sask)

Ctenocephalides spp.

Site: unspecified

Hosts: Felis catus (3)

unspecified $(1,2)$

Dist.: Canada, Ont, PEI

Record: 1. MacNay 1952 (Ont, PEI), 2. 1957

(Canada); 3. Slocombe 1973a (Ont)

Remarks: MacNay (1957) refered to

Ctenocephalides spp. as infesting household pets from coast to coast. 


\section{ORDER SIPHUNCULATA}

FAMILY HAEMATOPINIDAE

Haematopinus asini (Linnaeus, 1775)

Host: Equus caballus

Dist.: BC, Que

Records: Whitehead 1934 (Que); Spencer 1939 (BC), 1966 (BC)

Haematopinus eurysternus (Nitzsch, 1818)

Site: unspecified

Host: Bos taurus

Dist.: BC, NS, Ont, Que

Record: Thompson 1934 (NS, Que);

Whitehead 1934 (Que); Spencer 1939 (BC);

Teskey 1960 (Ont); Spencer 1966 (BC)

Haematopinus suis (Linnaeus, 1758) Leach, 1815

Site: unspecified

Host: Sus scrofa

Dist.: BC, Ont, Que

Records: Whitehead 1934 (Que); Spencer 1939

(BC); Rich 1952 (BC); Teskey 1960 (Ont);

Spencer 1966 (BC); Wright 1979 (Que)

Haematopinus spp.

Site: unspecified

Hosts: Bos taurus

Equus caballus

Dist.: Ont

Sus scrofa

Record: Slocombe 1973a

\section{FAMILY LINOGNATHIDAE}

Linognathus pedalis (Osborn, 1896)

Host: Ovis aries

Dist.: BC

Record: Spencer 1939

Linognathus setosus (Olfers, 1816)

Syn.: Linognathus piliferus (Burmeister)

Host: Canis familiaris

Dist.: BC, Man, Ont, Que

Records: Whitehead 1934 (Que); Spencer 1939

(BC); MacNay 1952 (Man, Ont); Judd 1953

(Ont)

Linognathus stenopsis (Burmeister, 1839) Enderlein, 1905

Site: unspecified

Host: Capra hircus

Dist.: Ont

Record: Teskey 1960

Linognathus vituli (Linnaeus, 1758) Enderlein, 1905

Site: unspecified

Host: Bos taurus

Dist.: Ont, Que

Record: Whitehead 1934 (Que); Teskey 1960

(Ont)
Linognathus spp.

Site: unspecified

Hosts: Bos taurus

Dist.: Ont

Canis familiaris

Records: Slocombe 1973a

Solenopotes capillatus Enderlein, 1904

Site: unspecified

Host: Bos taurus

Dist.: Ont

Record: Teskey 1960

Unidentified lice

Host: Bos taurus

Dist.: BC, Man

Record: MacNay 1957 


\section{HOST-PARASITE LIST}





\section{CLASS MAMMALIA}

\section{INFRACLASS EUTHERIA}

ORDER ARTIODACTYLA

\section{FAMILY BOVIDAE}

Bostaurus Ox

PROTOZOA

Coccidia gen. sp. (Ont)

Cryptosporidium spp. (Ont)

Eimeria alabamanensis (Ont)

E. arloingi (Ont)

E. aubernensis (Ont)

E. bovis (Ont)

E. bukidnonensis (Ont)

E. canadensis (Ont)

E. cylindrica (Ont)

E. ellipsodalis (Ont)

E. wyomingensis (Ont)

E. zuernii (Ont)

Sarcocystis spp (Ont)

Trichomonas spp. (Ont)

Trypanosoma rutherfordi (BC)

T. theileri (Ont)

T. uniforme (Ont)

Trypanosoma spp. (BC)

\section{TREMATODA}

Calicophoron microbothrioides (Que)

Dicrocoelium dendriticum (BC, Nfld)

Fascioloides magna (Alta, Ont)

Paramphistomum liorchis (Que)

Paramphistomum spp. (Alta, Ont)

\section{CESTOIDEA}

Cysticercus bovis (Ont, Sask)

C. tenuicollis (Ont, Que)

Moniezia benedeni egg (NB, Que)

M. expansa (Que)

Moniezia spp. (Ont, Que)

\section{NEMATODA}

Ascaris lumbricoides larva (Ont)

Bunostomum phlebotomum (Que)

B. trigonocephalum (Ont, Que)

Bunostomum spp. (Ont, Que)

Capillaria spp. (Ont, Que)

Cooperia oncophora (NB, Nfld)

C. punctata (Ont)

Cooperia spp. (Ont, Que)

Dictyocaulus viviparus (Alta, Nfld, Ont, Que)

Dictyocaulus spp. (Ont)

Haemonchus contortus (Ont, Prairie

Provinces, Que)

Haemonchus spp. (Ont)

Nematoda gen. sp. (Que)

Nematodirus helvetianus (NB, Ont)
Nematodirus spp. (Ont, Que)

Oesophagostomum radiatum (NB, Nfld, Ont, Que)

Oesophagostomum spp. (Ont, Que)

Onchocerca gutturosa (Ont, Que)

O. lienalis (Ont, Que)

Onchocerca spp. (Man, Sask)

Ostertagia circumcincta (Ont)

O. lyrata (Ont)

O. ostertagi (NB, Ont, Que)

O. trifurcata (Alta, Ont)

Ostertagia spp. (Ont, Que)

Parafilaria bovicola (Alta, Que)

Setaria labiatopapillosa (Alta, Man, Ont,

Que)

Stephanofilaria stilesi (Alta)

Strongyloidea gen. sp. (Ont)

Strongyloides papillosus (Que)

Strongyloides spp. (Ont)

Thelazia lacrymalis (Ont)

T. gulosa (Ont)

Trichostrongyloidea gen. sp. egg (Ont)

Trichostrongylus axei (NB, Ont, Que)

Trichostrongylus spp. (Ont)

Trichuridae gen. sp. (Ont)

Trichuris ovis (NB, Que)

Trichuris spp. (Alta, NB, NWT, Ont, Sask)

Trichuris spp. egg (NB, Ont)

Unidentified Rhabditoidea (Ont)

\section{CHELICERATA}

\section{ACARI}

Chorioptes bovis (Ont)

Demodex bovis (Ont)

Demodex spp. (Ont)

Dermacentor andersoni (Alta, BC, Sask)

D. albipictus (BC, NB, NS, Sask)

Haemaphysalis cinnabarina (Man)

Ixodes cookei (NB, Ont, Que)

Otobius megnini $(\mathrm{BC})$

Sarcoptes scabiei (Ont)

\section{UNIRAMIA}

\section{DIPTERA}

Hypoderma bovis larva (Alta, BC, Ont)

H. lineatum (Alta, BC)

Hypoderma spp. (Ont, Sask)

Phormia regina $(\mathrm{BC})$

\section{MALLOPHAGA}

Damalinia bovis (BC, Man, NS, Ont, Que)

Damalinia spp. (Ont)

\section{SIPHUNCULATA}

Haematopinus eurysternus (BC, NS, Ont, Que) 
Haematopinus spp. (Ont)

Lice (unidentified) (BC, Man)

Linognathus vituli (Ont, Que)

Linognathus spp. (Ont)

Solenopotes capillatus (Ont)

Caprahircus Domestic Goat

UNIRAMIA

MALLOPHAGA

Damalinia caprae (BC)

D. limbata (Ont)

SIPHUNCULATA

Linognathus stenopsis (Ont)

Ovis aries Domestic Sheep

PROTOZOA

Coccidia gen. sp. (Que, Sask)

Eimeria ahsata (Alta)

E. arloingi (Alta, Ont)

E. crandallis (Alta)

E. faurei (Alta)

E. granulosa (Alta)

E. intricata (Alta)

E. ninakohlyakimovae (Alta, Ont)

E. pallida (Alta)

E. parva (Alta, Ont)

E. punctata (Alta)

Eimeria spp. (Que)

Sarcocystis spp. (Sask)

Toxoplasma spp. (Ont)

\section{TREMATODA}

Dicrocoelium dendriticum (NS, Que)

Fasciola hepatica (Canada)

\section{CESTOIDEA}

Cysticercus ovis (Canada, Man, NS)

C. tenuicollis (Alta, Canada, Ont, Que)

Moniezia expansa (Canada, Nfld, NS, Ont,

Que)

Moniezia spp. (Nfld, Sask)

Taenia hydatigena cysticercus (Que, Sask)

Thysanosoma actinioides (Alta, BC, Canada,

Sask)

Thysanosoma spp. (Ont, Sask)

\section{NEMATODA}

Ascaris suum (Que)

Ascaris spp. (Canada, Ont)

Bunostomum trigonocephalum (Canada,

Nfld, NS, Ont, Que)

Bunostomum spp. (Que, Sask)

Capillaria longipes (Canada, Que)

Capillaria spp. (NS, Ont, Que)

Chabertia ovina (Canada, NS, Ont, Que)

Chabertia spp. (Ont, Sask)

Cooperia curticei (Canada, NS, Ont, Que)

C. oncophora (Canada, NS, Ont)
Cooperia spp. (Ont, Que, Sask)

Dictyocaulus filaria (Alta, Canada, Nfld, Ont, PEI, Que)

Dictyocaulus spp. (Ont)

Gongylonema pulchrum (Canada, Ont)

Haemonchus contortus (Canada, NS, Ont,

Que)

Haemonchus spp. (Ont, Que, Sask)

Muellerius capillaris (Canada, Ont)

Muellerius spp. (Ont)

Nematodirus abnormalis (Nfld)

$N$. filicollis (Canada, NS, Ont, Que)

$N$. helvetianus (NB, Ont)

$N$. spathiger (Canada, Nfld, NS, Ont, Que)

Nematodirus spp. (Ont, Que, Sask)

Oesophagostomum columbianum (Canada, NS, Ont, Que)

$O$. venulosum (Canada, Nfld, NS, Ont, Que)

Oesophagostomum spp. (Nfld, Ont, Que)

Ostertagia circumcincta (Canada, Nfld, NS,

Ont, Que)

O. trifurcata (NS)

Ostertagia spp. (Ont, Que, Sask)

Parelaphostrongylus tenuis exp. (Ont)

Protostrongylus spp. (Ont)

Skrjabinema spp. (Sask)

Strongyloides papillosus exp. (Canada, NS,

Ont, Que)

Strongyloides spp. (Ont, Que, Sask)

Telodorsagia davtiani (NS)

Trichostrongylus axei (Canada, NS, Ont,

Que)

T. colubriformis (Canada, NS, Ont, Que)

$T$. vitrinus (Canada, NS, Ont, Que)

Trichostrongylus spp. (Ont, Que, Sask)

Trichuris ovis (Canada, Nfld, NB, NS, Ont, Que)

Trichuris spp. (Alta, NWT, Ont, Sask)

\section{CHELICERATA}

ACARINA

Dermacentor andersoni (Alta, BC)

Otobius megnini (BC)

\section{UNIRAMIA}

DIPTERA

Lucilia illustris (Ont)

L. sericata $(\mathrm{Nfld})$

Melophagus ovinus (BC, Ont, Sask)

Oestrus ovis (Ont, Western Canada)

Phormia regina (Ont)

P. terranovae (Ont)

Unidentified Diptera (BC)

SIPHUNCULATA

Linognathus pedalis (BC) 
FAMILY SUIDAE

Sus scrofa Domestic Pig

PROTOZOA

Balantidium spp. (Ont)

Eimeria dublieki (Ont)

Toxoplasma spp. (Ont)

Trichomonas spp. (Ont)

\section{TREMATODA}

Paragonimus spp. (Ont)

\section{CESTOIDEA}

Cysticercus cellulosae (Man, Ont, Sask)

Echinococcus granulosus hydatid cyst (Ont)

Echinococcus spp. hydatid (Ont)

Multiceps spp. (Ont)

Taenia hydatigena cysticercus (Que)

\section{NEMATODA}

Ascaris lumbricoides larva (All Provinces,

Ont)

A. suum (Nfld, Que, Sask)

Ascaris spp. (Ont)

Ascarops spp. (Ont)

Hyostrongylus rubidus (Ont, Que)

Hyostrongylus spp. (Ont)

Metastrongylus pudendotectus (Ont)

M. salmi (NS, Ont, PEI, Que)

Metastrongylus spp. (Ont)

Oesophagostomum dentatum (NB, Ont, Que)

O. quadrispinulatum (NB)

Oesophagostomum spp. (NB, Ont, Que)

Ollulanus tricuspis (Ont)

Physocephalus spp. (Ont)

Stephanurus dentatus (Man, NS)

Strongyloides spp. (Ont)

Trichinella spiralis cyst/ larva (BC, Man, NB,

NS, Ont, PEI, Que)

Trichuris suis (Ont, Que)

Trichuris spp. (Ont)

\section{ACANTHOCEPHALA}

Macracanthorhynchus hirudinaceus (Ont)

\section{CHELICERATA}

ACARI

Demodex phylloides (Ont)

Dermacentor andersoni (BC)

Sarcoptes scabiei (Ont)

UNIRAMIA

SIPHUNCULATA

Haematopinus suis (BC, Ont, Que)

Haematopinus spp. (Ont)

\section{ORDER CARNIVORA}

FAMILY CANIDAE

\author{
Canis familiaris Domestic Dog \\ PROTOZOA \\ Eimeria canis (Ont, Que) \\ Endolimax nana (Sask) \\ Entamoeba blattae (Que) \\ E. coli (Que, Sask) \\ E. histolytica (Que, Sask) \\ Giardia canis (Que) \\ Giardia spp. (Ont) \\ Isospora bigemina (Que) \\ I. felis (Ont, Que) \\ I. rivolta (Ont, Que) \\ Isospora spp. (Alta, NB, NWT, Que, Sask) \\ Toxoplasma gondii (Ont) \\ Toxoplasma spp. (Ont) \\ Trichomonas spp. (Ont)
}

TREMATODA

Alaria arisaemoides (Sask)

A. canis (Ont)

Alaria spp. (Alta, NWT, Ont, Que, Sask)

Cryptocotyle lingua (Nfld, Que)

Metorchis conjunctus (Alta, Man, NWT,

Ont, Que, Sask)

Metorchis spp. (Sask)

Paragonimus kellicotti (Ont)

Paragonimus spp. (Ont)

Parametorchis complexus (Man)

Unidentified Trematoda (Man, NWT, Que)

\section{CESTOIDEA}

Diphyllobothrium latum (Alta, Nfld, NWT, Que, Sask)

Diphyllobothrium spp. (Alta, Man, Nfld,

NWT, Ont, Que, Sask)

Dipylidium caninum (NB, Nfld, NWT, Ont)

Dipylidium spp. (Ont, Sask)

Echinococcus granulosus (Ont, Que)

Taenia crassiceps (Ont)

T. elliptica (Que)

T. hydatigena (Nfld, Que)

T. pisiformis (Alta, Nfld, NWT, Ont, Que)

T. serialis (Alta, NWT)

Taenia spp. (BC, Man, NWT, Ont, Que,

Sask)

Taenia/Echinococcus spp. (egg) (Alta, NWT, Sask)

Unidentified Cestoidea (Man, Que)

NEMATODA

Ancylostomatidae gen. sp. (Alta, NB, NWT, Que, Sask)

Ancylostomum caninum (Alta, Ont, Que)

Ancylostomum spp. (Ont, Sask)

Ascaris spp. (Que)

Capillaria aerophila (Que)

Capillaria spp. (Ont) 
Corynosoma semerme (NWT)

Crenosoma vulpis (Nfld)

Dioctophyma renale (Man, NWT, Ont, Que)

Dipetalonema spp. (Ont)

Dirofilaria immitis (Alta, Ont)

Dirofilaria spp. (Ont)

Dracunculus insignis (Que)

Filaroides milksi $(\mathrm{BC})$

Filaroides spp. (Ont)

Oslerus osleri (Que)

Pelodera strongyloides (Ont)

Physaloptera rara (Que)

Spirocerca spp. (Ont)

Strongyloides stercoralis (Que)

Strongyloides spp. (Ont)

Toxascaris leonina (Alta, Man, NB, Nfld,

NWT, Ont, Que, Sask)

Toxascaris spp. (Ont, Sask)

Toxocara canis (Alta, Man, NB, Nfld, NS,

NWT, Ont, Que, Sask)

Toxocara spp. (Ont, Sask)

Trichuris vulpis (Nfld, Ont, Que)

Trichuris spp. (Alta, NWT, Ont, Sask)

Trichuris spp. egg (NB, Que, Sask)

Uncinaria stenocephala (Alta, BC, Lab, Man,

Nfld, NWT, Ont, Que)

Uncinaria spp. (Ont, Sask)

Unidentified Rhabditoidea (Ont)

\section{CHELICERATA}

\section{ACARI}

Cheyletiella spp. (Ont)

Demodex spp. (Ont)

Dermacentor albipictus (Sask)

D. andersoni (BC)

D. variabilis (Man, Ont)

Ixodes cookei (BC, NB, Ont, Que)

I. ricinus $(\mathrm{BC})$

I. ricinus var. scapularis $(\mathrm{BC})$

Ixodes spp. (Ont)

Notoedres spp. (Ont)

Otobius spp. (Ont)

Otodectes cynotis (Que)

O. cynotis var. canis $(\mathrm{BC})$

Pneumonyssus spp. (Ont)

Rhipicephalus sanguineus (BC, NS, Ont)

Rhipicephalus spp. (Ont)

Sarcoptes scabiei (Ont)

Trombiculidae gen. sp. (Ont)

\section{UNIRAMIA}

DIPTERA

Cuterebra spp. (Ont)

MALLOPHAGA

Trichodectes canis $(\mathrm{BC})$
Trichodectes spp. (Ont)

\section{SIPHONAPTERA}

Ctenocephalides canis (Alta, BC, NB, Ont)

C. felis (BC, NS)

Ctenocephalides felis felis (BC, Man, NB,

Nfld, NS, Ont, Que, Sask)

Tamiophila grandis (NB)

SIPHUNCULATA

Linognathus setosus (BC, Man, Ont, Que)

Linognathus spp. (Ont)

\section{FAMILY FELIDAE}

Felis catus Domestic Cat PROTOZOA

Isospora felis (Ont)

I. rivolta (Ont)

Isospora spp. (NB)

Toxoplasma gondii (Ont)

Toxoplasma spp. (Ont)

TREMATODA

Metorchis conjunctus (Ont, Que)

Parametorchis complexus (Alta)

Plagiorchis massino (Nfld)

\section{CESTOIDEA}

Diphyllobothrium dendriticum (Nfld)

Diphyllobothrium spp. (Ont)

Dipylidium caninum (Nfld, Ont)

Dipylidium spp. (Ont)

Echinococcus multilocularis (Sask)

Taenia taeniaeformis (Nfld, Que)

Taenia spp. (Ont)

NEMATODA

Aelurostrongylus spp. (Ont)

Ancylostomum spp. (Ont)

Capillaria hepatica (NB)

Capillaria spp. (Ont)

Dirofilaria immitis (Ont)

Ollulanus tricuspis (Alta, Ont, Que)

Rhabditoidea gen. sp. (Ont)

Strongyloides spp. (Ont)

Toxascaris leonina (NB, Nfld)

Toxascaris spp. (Ont)

Toxascaris cati (NB, Nfld, NS, Que)

Toxocara spp. (Ont)

\section{CHELICERATA}

ACARI

Dermacentor andersoni (BC)

Dermacentor spp. (Ont)

Haemophysalis leporis-palustris (BC)

Ixodes cookei (NB, Ont, Que)

Ixodes spp. (Ont) 
Otobius lagophilus (Alta)

O. megnini (BC)

Otodectes cynotis (BC, NB, Que)

O. cynotis var. felis $(\mathrm{BC})$

Sarcoptes scabiei (unspecified)

\section{UNIRAMIA}

DIPTERA

Calliphoridae gen. sp. (Ont)

Cuterebra spp. (Ont)

\section{MALLOPHAGA}

Felicola subrostratus (BC, Que)

\section{SIPHONAPTERA}

Ctenocephalides canis (Alta, BC)

C. f. felis (BC, NS)

C. felis (Alta, BC, Man, Nfld, NB, NS, Ont,

Que, Sask)

Ctenocephalides spp. (Ont)

Monopsyllus vison (Sask)

\section{ORDER PERISSODACTYLA}

\section{FAMILY EQUIDAE}

\section{Equus caballus Domestic Horse \\ PROTOZOA \\ Cryptosporidium spp. (Sask) \\ Eimeria crandallis (Ont) \\ E. granulosa (Ont) \\ E. intricata (Ont) \\ E. leuckarti (Ont) \\ Klossiella equi (NB) \\ Sarcocystis spp. (Ont) \\ Toxoplasma spp. (Ont, Sask) \\ Trichomonas spp. (Ont) \\ Trypanosoma equiperdum (Alta)}

\section{CESTOIDEA}

Anoplocephala magna (Ont, Que)

A. perfoliata (Nfld, Ont)

Anoplocephala spp. (Ont)

Paranoplocephala mamillana (BC, Que, Sask)

\section{NEMATODA}

Capillaria hepatica (Alta)

Citellinema bifurcatum (Sask)

Cyathostoma spp. (Nfld)

Cyathostomum catinatum (Alta, Nfld, Sask)

C. coronatum (Sask)

C. labiatum (Alta, Sask)

C. labratum (Alta, Sask)

Cylicocyclus elongatus (Nfld, Sask)

C. insigne (Alta)

C. nassatus (Alta, Nfld, Sask)

C. radiatus (Alta, Sask)

Cylicodontophorus bicornatus (Alta, Sask)
Cylicostephanus calicatus (Alta, Sask)

C. goldi (Alta, Sask)

C. poculatus (Alta, Nfld, Sask)

Dictyocaulus arnfieldi (Ont)

Dictyocaulus spp. (Ont)

Gyalocephalus capitatus (Alta, NB, Ont, Sask)

Gyalocephalus spp. (NB)

Habronema majus (Ont)

H. muscae (Ont)

Habronema spp. (Ont)

Nematoda gen. sp. (Nfld)

Nematodirus spathiger (Que)

Oesophagodontus robustus (Alta, Nfld, Ont, Que, Sask)

Oesophagodontus spp. (NB)

Onchocerca cervicalis (BC, Que)

Oxyuris equi (NB, Nfld, Ont, Que)

Oxyuris spp. (Ont)

Parascaris equorum (NB, Nfld, Ont, Que)

Parascaris spp. (Ont)

Poteriostomum spp. (NB)

Probstmayria vivipara (NB, Que)

Setaria equina (Ont, Que)

Setaria spp. (Ont)

Strongyles gen. sp. (NB, Ont, Que)

Strongyloides spp. (Ont)

Strongylus edentatus (Alta, NB, Nfld, Ont,

Que, Sask)

S. equinus (Alta, NB, Ont, Que, Sask)

S. vulgaris (Alta, NB, Nfld, Ont, Que, Sask)

Strongylus spp. (NB, Ont)

Thelazia lacrymalis (Ont)

Thelazia spp. (Ont)

Triodontophorus brevicauda (Alta, Que,

Sask)

T. minor (Nfld)

T. serratus (Alta, Nfld, Ont, Que, Sask)

T. tenuicollis (Alta, Sask)

Triodontophorus spp. (NB, Ont)

\section{CHELICERATA}

ACARI

Chorioptes spp. (Ont)

Dermacentor albipictus (Alta, BC, NB, NS,

Sask)

D. andersoni (Alta, BC)

Dermacentor spp. (Ont)

Psoroptes spp. (Ont)

UNIRAMIA

DIPTERA

Gasterophilus intestinalis (Alta, Ont)

G. nasalis (Alta, Ont)

Gasterophilus spp. (Man)

Hypoderma spp. (Ont)

\section{MALLOPHAGA}

Damalinia equi (BC) 
Damalinia spp. (Ont)

Trichodectes equi $(\mathrm{BC})$

\section{SIPHUNCULATA}

Haematopinus asini (BC, Que)

Haematopinus spp. (Ont) 


\section{References}

ALLEN, G.W. 1962. Acute atypical bovine pneumonia caused by Ascaris lumbricoides. Canadian Journal of Comparative Medicine and Veterinary Science 26: 241-243.

ALLEN, J.A., AND R.A. WARDLE. 1934. Fluke disease in northern Manitoba sledge dogs. Canadian Journal of Research 10: 404-408.

ALLEN, J.R., AND J.H.L. MILLS. 1971. Alaria arisaemoides in Saskatchewan dogs. Canadian Veterinary Journal 12: 24-28.

AMIN, O.M. 1982. Acanthocephala. pp. 933-940. In. Synopsis and Classification of Living Organisms. McGraw-Hill Book Co., New York.

ANDERSON, R.C., A.G. CHABAUD, AND S. WILLMOTT, eds. 1974-1983. CIH Keys to the Nematode Parasites of Vertebrates Nos. 1-10. Commonwealth Agricultural Bureaux, Farnham Royal, Bucks, England.

ANDERSON, R.C., AND U.R. STRELIVE. 1966. Experimental cerebrospinal nematodiasis (Pneumostrongylus tenuis) in sheep. Canadian Journal of Zoology 44: 889-894.

ANVIK, J.O., A.E. HAGUE, AND A. RAHAMAN. 1974. A method of estimating urban dog populations and its application to the assessment of canine fecal pollution and endoparasitism in Saskatchewan. Canadian Veterinary Journal 15: 219-223.

AUSTIN, R.J., AND K.H. DIES. 1981. Klossiella equi in the kidneys of a horse. Canadian Veterinary Journal 22: 159-161.

AXELSON, R.D. 1962. Metorchis conjunctus liver fluke infestation in a cat. Canadian Veterinary Journal 3: 359-360.

BARKER, I.K. 1970. Thelazia lacrymalis from the eyes of an Ontario horse. Canadian Veterinary Journal 11: 186-189.

BASIR, M.A. 1950. The morphology and development of the sheep nematode, Strongyloides papillosus (Wedl, 1856). Canadian Journal of Research 28: 173-196.

BEQUAERT, J.C. 1945. The ticks, or Ixodoidea, of the northeastern United States and eastern Canada.

Entomologica Americana 25: 121-224.

BERNARD, M.A. 1970. Feline dirofilariasis. Canadian Veterinary Journal 11: 190-191.

1971. Dirofilariasis in Canada. Canadian Veterinary Journal 12: 139.

BLAKLEY, B.R. 1979. A livestock disease survey from auction mart inspection in Saskatchewan. Canadian Veterinary Journal 20: 358-360.

BLITZ, N.M., AND H.C. GIBBS. 1971. Morphological characterization of the stage of arrested development of Haemonchus contortus in sheep. Canadian Journal of Zoology 49: 991-995.

BOUVRY, M., AND M.E. RAU. 1984. Paramphistomum spp. in dairy cattle in Quebec. Canadian Veterinary Journal 25: 353-356.

BROOKS, D.R., R.T. O'GRADY, AND D.R. GLEN. 1985. Phylogenetic analysis of the Digenea (Platyhelminthes: Cercomeria) with comments on their adaptive radiation. Canadian Journal of Zoology 63: 411-443.

BROWN, J.H. 1944a. The fleas (Siphonaptera) of Alberta, with a list of the known vectors of sylvatic plague. Annals of the Entomological Society of America 37: 207-213

1944b. The spotted fever and other Albertan ticks. Canadian Journal of Research 22: 36-51.

BROWN, J.H., AND G.M. KOHLS. 1950. The ticks of Alberta with special reference to distribution. Canadian Journal of Research 28: 197-205.

BROWN, N.R. 1955. Additional host and distribution records of some eastern Canadian Siphonaptera. Canadian Entomologist 87: 362-365.

1968. Notes on the Siphonaptera of New Brunswick. Canadian Entomologist 100: 486-498.

BUCKNER, C.H. 1964. Fleas (Siphonaptera) of Manitoba mammals. Canadian Entomologist 96: 850-856.

CALDWELL, D. 1984. Ollulanus tricuspis in an Ontario cattery. Veterinary Bulletin 54: 314-315.

CAMERON, A.E., AND J.S. FULTON. 1927. A local outbreak of the winter or moose tick, Dermacentor albipictus, Pack. ( Ixodoidea) in Saskatchewan. Bulletin of Entomological Research 17: 249-257.

CAMERON, T.W.M. 1938. Investigations on trichinosis in Canada. I. A preliminary survey of the incidence of Trichinella spiralis in hogs in eastern Canada. Canadian Journal of Research 16: 89-92.

1939. Investigations on trichinosis in Canada. II. A further survey of the incidence of Trichinella spiralis in hogs in eastern Canada. Canadian Journal of Research 17: 151-153.

1940a. Investigations on trichinosis in Canada. III. On the incidence of trichinosis in garbage-fed hogs. Canadian Journal of Research 18: 83-85.

1940b. Ollulanus tricuspis in the cat in Canada. Canadian Journal of Comparative Medicine 4: 296-297.

CAMERON, T.W.M., I.W. PARNELL, AND L.L. LYSTER. 1940. The helminth parasites of sledge-dogs in Northern Canada and Newfoundland. Canadian Journal of Research 18: 325-332.

CAMPBELL, D.J., D.L. DIAMOND, AND A.A. KINGSCOTE. 1960. Nematodiriasis in calves. Canadian Veterinary Journal 1: 119-122.

CAMPBELL, D.J., AND G.D. WETHERILL. 1957. Parasitic bronchitis in adult cattle in Ontario - a case report. Journal of the American Veterinary Medical Association 131: 273-275.

CHOQUETTE, L.P.E. 1945. Observation on the incidence 
of the common stomach worm, Haemonchus contorius, in young cattle in a Montreal abattoir. Canadian Journal of Comparative Medicine 9: 330-331.

1956. Observations on experimental infection of dogs with Echinococcus. Canadian Journal of Zoology 34: 190-192.

CHOQUETTE, L.P.E., AND L. de G. GELINAS. 1950. The incidence of intestinal nematodes and protozoa in dogs in the Montreal district. Canadian Journal of Comparative Medicine 14: 33-38.

CLARK, E.G., H.G.G. TOWNSEND, AND N.T. McKENZIE. 1981. Equine protozoal myeloencephalitis: A report of two cases from western Canada. Canadian Veterinary Journal 22: 140-144.

COMFORT, C.M., AND R.D. AXELSON. 1962. Two reports of unusual parasites diagnosed in dogs. Canadian Veterinary Journal 3: 22-24.

CONKLIN, R.L., AND A.D. BAKER. 1930. Presence of the lancet fluke, Dicrocoelium dendriticum (Rudolphi, 1819), in Canada. Journal of Parasitology 17: 18-19.

COOLEY, R.A., AND G.M. KOHLS. 1945. The genus Ixodes in North America. U.S. Public Health Service, National Institute of Health Bulletin No. 184, 246 pp.

DAVENPORT, P.G., AND P.H.G. STOCKDALE. 1967. A case of hyostrongylosis in Ontario. Canadian Veterinary Journal 8: 288-290.

DEROTH, L., AND J.L. FRECHETTE. 1975. Dracunculus insignis (Leidy, 1858) chez un berger allemand. Canadian Veterinary Journal 16: 176-178.

DIES, K.H., AND J. PRITCHARD. 1985. Bovine Stephanofilarial dermatitis in Alberta. Canadian Veterinary Journal 26: 361-362.

EMERSON, K.C. 1972. Checklist of the Mallophaga of North America (North of Mexico). Deseret Test Center. Dugway, Utah.

FALLIS, A.M. 1938. A study of the helminth parasites of lambs in Ontario. Transactions of the Royal Canadian Institute 22: 81-128.

1940. Studies on Oestrus ovis L. Canadian Journal of Research 18: 442-446.

FLETCH, A.L. 1969. Pneumothorax in a dog with Dirofilaria immitis infection. Canadian Veterinary Journal 10: 80-82.

FRANK, J.F. 1952. A study of the incidence of Trichinosis in swine in the maritime provinces. Canadian Journal of Comparative Medicine 16: 73-77.

FRECHETTE, J.L., AND H.C. GIBBS. 1971. Studies on the incidence of gastrointestinal helminths of cattle in Quebec. Canadian Veterinary Journal 12: 207-210.

FREEMAN, R.S. 1962. Studies on the biology of Taenia crassiceps (Zeder, 1800) Rudolphi, 1810 (Cestoda). Canadian Journal of Zoology 40: 969-990.

FREEMAN, R.S., A. ADORJAN, AND D.H. PIMLOTT. 1961. Cestodes of wolves, coyotes, and coyote-dog hybrids in Ontario. Canadian Journal of
Zoology 39: 527-532.

FRIMETH, J.P., AND H.P. ARAI. 1983. Some potential mosquito vectors of the canine heartworm, Dirofilaria immitis, in the Calgary region of southern Alberta. Canadian Journal of Zoology 61: 1156-1158.

GAJADHAR, A.A., J.P. CARON, AND J.R. ALLEN. 1985. Cryptosporidiosis in two foals. Canadian Veterinary Journal 26: 132-134.

GHADIRIAN, E., P. VIENS, H. STRYKOWSKI, AND F. DUBREUIL. 1976. Epidemiology of toxocariasis in the Montreal area. Canadian Journal of Public Health 67: 495-498.

GIBBS, H.C. 1964. Observations on an outbreak of clinical parasitism in ewes during the winter months. Canadian Veterinary Journal 5: 8-11.

GILLICK, A. 1972. Paragonimiasis in a dog. Canadian Veterinary Journal 13: 175-179.

GRAESSER, F.E. 1957. Lungworm disease of cattle in Alberta. Canadian Journal of Comparative Medicine 21: $355-358$.

GREENWAY, J.A., AND P.H.G. STOCKDALE. 1970. A case tentatively diagnosed as Filaroides milksi in a dog. Canadian Veterinary Journal 11: 203-204.

GREGSON, J.D. 1935. The production of artificial conditions conducive to winter feeding of ticks. Proceedings of the B.C. Entomological Society (1935): 6-7.

1937. Notes on some phenomenal feeding in ticks. Proceedings of the B.C. Entomological Society (1937): 8-11.

1947. Feeding periods prerequisite to the mating of Dermacentor andersoni. Proceedings of the B.C. Entomological Society (1946) 43: 3-6.

1952. Further studies on tick paralysis. Proceedings of the B.C. Entomological Society 48: 54-58.

1953. Records of the tick Otobius megnini (Duges) from British Columbia (Acarina: Argasidae). Proceedings of the B.C. Entomological Society 49: 30.

1958a. Host susceptibility to paralysis by the tick Dermacentor andersoni Stiles (Acarina: Ixodidae). Canadian Entomologist 90: 421-424.

1958b. Recent cattle grub life-history studies at Kamloops, British Columbia, and Lethbridge, Alberta. 10th International Congress of Entomology 3, (1958): 725-734.

1958c. Tick paralysis in cattle in British Columbia in 1957. Proceedings of the B.C. Entomological Society 55: 6-7.

GRIFFITHS, H.J. 1937. Some observations on the overwintering of certain helminth parasites of sheep in Canada. Canadian Journal of Research 15: 156-162.

HADWEN, S. 1913. On "Tick Paralysis" in sheep and man following bites of Dermacentor venustus, with notes on the biology of the tick. Parasitology 6: 283-297.

HALL, R.R., AND McKIEL, J.A. 1961. Occurrences of 
the American dog tick, Dermacentor variabilis (Say) in western Nova Scotia. Canadian Entomologist 93: 891-893.

HARVEY, R.V. 1907. British Columbia fleas. Bulletin of the Entomological Society of British Columbia No. 7.

HEARLE, E. 1933. Notes on a serious outbreak of tick paralysis in cattle. Proceedings of the B.C.

Entomological Society (1933): 11-16.

1938. The ticks of British Columbia. Scientific Agriculture 18: 341-354.

HEWITT, C.G. 1915. A contribution to a knowledge of Canadian ticks. Transactions of the Royal Society of Canada 9(series 3): 225-239.

HOLLAND, G.P. 1940. Notes on the ecology of Dermacentor andersoni in southern Alberta.

Proceedings of the B.C. Entomological Society 36: 11-12.

1984. The fleas of Canada, Alaska and Greenland (Siphonaptera). Memoirs of the Entomological Society of Canada No. 130. 631 pp.

HOPKINS, G.H.E. 1960. Notes on some Mallophaga from mammals. Bulletin of the British Museum of Natural History (Entomology) 10: 77-98.

HULLAND, T.J. 1956. Toxoplasmosis in Canada. Journal of the American Veterinary Medical Association 128: 74-79.

JARVIS, T.D. 1910. The Acarina, with a host index to the species found in Ontario. 40th Annual Report of the Entomological Society of Ontario 36: 82-109.

JOHNSTON, E.F., AND L.W. MacPHERSON. 1956. An outbreak of acute parasitic gastroenteritis in a herd of cattle in the Ottawa Valley. Canadian Journal of Comparative Medicine 20: 203-205.

JUDD, W.W. 1953. Mammal host records of Acarina and Insecta from the vicinity of London, Ontario. Journal of Mammalogy 34: 137-139.

1954. Some records of ectoparasitic Acarina and Insecta from mammals in Ontario. Journal of Parasitology 40: 483-484.

KENNEDY, M.J. 1983. A revision of North American species of the Genus Parametorchis Skrjabin, 1913 (Trematoda: Opisthorchiidae). Proceedings of the Helminthological Society of Washington 50: 312-317.

KENNEDY, M.J., R.A. KRALKA, AND M. SCHOONDER WOERD. 1984. First report of Ollulanus tricuspis (Nematoda) from western Canada. Journal of Parasitology 70: 319-320.

KRANTZ, G.W. 1978. A manual of Acarology. 2nd ed. Oregon University Book Store Inc. Corvallis. 509 pp.

LaRUE, G.R., AND A.M. FALLIS. 1934. Alaria canis n. sp. (Alaridae), a trematode parasite of the dog in northern Ontario. Journal of Parasitology 20: 335.

LEES, M.J., N. KLEIDER, AND T.J. TUDDENHAM. 1983. Cutaneous onchocerciasis in the horse: five cases in southwestern British Columbia. Canadian Veterinary
Journal 24: 3-5.

LEVINE, N.D. (Chairman). 1980. A newly Revised Classification of the Protozoa. By The Committee on Systematics and Evolution of the Society of Protozoologists. Journal of Protozoology 27: 37-58.

LEWIS, JR., P.D. 1974. Presence of Dicrocoelium dendriticum (Rudolphi, 1819) (Trematoda:

Dicrocoeliidae) in western Canada. Canadian Journal of Zoology 52: 662-663.

LITTLE, P.B. 1972. Cerebrospinal nematodiasis of Equidae. Journal of the American Veterinary Medical Association 160: 1407-1413.

LORD, L.H., AND J.K. McGREGOR. 1960. A case of Strongyloides dermatitis in Canada. Canadian Veterinary Journal 1: 111-114.

MACNAY, C.G. 1952. Summary of the more important insect infestations and occurrences in Canada in 1951. 82nd Annual Report of the Entomological Society of Ontario (1951). pp. 91-115.

1957. Summary of important insect infestations, occurrences and damage in Canada in 1957. Annual Report of the Entomological Society of Ontario (1957) 88: 63-78.

MADDY, K.T. 1955. Stephanofilarial dermatitis in cattle. The North American Veterinarian 36: 275-280.

MAHRT, J.J. 1969. Prevalence of coccidia in domestic sheep in central Alberta. Canadian Veterinary Journal 10: $176-178$.

MALLOY, W.F., AND J.A. EMBIL. 1973. Prevalence of Toxocara sp. in dogs and cats in Halifax. Canadian Journal of Public Health 64: 84.

1978. Prevalence of Toxocara spp. and parasites in dogs and cats in Halifax, Nova Scotia. Canadian Journal of Comparative Medicine 42: 29-31.

MARCOUX, M., J.L. FRECHETTE, AND M. MORIN. 1977. Infection par Onchocerca cervicalis au Quebec: signes cliniques et methode de diagnostic. Canadian Veterinary Journal 18: 108-110.

MARGOLIS, L., AND J.R. ARTHUR. 1979. Synopsis of the Parasites of Fishes of Canada. Bulletin of the Fisheries Research Board of Canada No. 199. 269 pp.

MARTIN, L.J., H.C. GIBBS, AND J.W. PULLIN. 1974. Gastrointestinal parasites of swine in Quebec. I. An incidence survey. Canadian Veterinary Journal 15: 72-76.

McGREGOR, J.K., AND A.A. KINGSCOTE. 1957. A survey of gastro-intestinal helminths of cattle in Ontario. Canadian Journal of Comparative Medicine 21: 370-373.

McLEOD, J.A. 1967. Dioctophyma renale infections in Manitoba. Canadian Journal of Zoology 45: 505-508.

MONGEAU, N. 1961. Hepatic distomatosis and infectious canine hepatitis in northern Manitoba. Canadian Veterinary Journal 2: 33-38.

MOOLENBEEK, W.J., AND G.A. SURGEONER. 1980. 
Southern Ontario survey of eyeworms, Thelazia gulosa and Thelazia lacrymalis in cattle and larvae of Thelazia spp. in the face fly, Musca autumnalis. Canadian Veterinary Journal 21: 50-52.

MOTEANE, M., D.M. MIDDLETON, AND L.R. POLLEY. 1979. A survey of disease conditions in adult and feeder sheep in Saskatchewan. Canadian Veterinary Journal 20: 2-7.

MOYNIHAN, I.W., AND I.W. MUSFELDT. 1949. A study of the incidence of trichinosis in swine in British Columbia. Canadian Journal of Comparative Medicine 13: 224-227.

NATION, P.N., AND K.H. DIES. 1978. Capillaria hepatica in a horse. Canadian Veterinary Journal 19: 315-316.

NELSON, W.A. 1952. A note on the presence of first-stage larvae of Gasterophilus intestinalis (Degeer) (Diptera: Gasterophilidae) in the mouth of the horse. Canadian Entomologist 84: 356.

NELSON, W.A., AND J. WEINTRAUB. 1972. Hypoderma lineatum (DeVill) (Diptera: Oestridae): Invasion of the bovine skin by newly hatched larvae. Journal of Parasitology 58: 614-624.

NIELSEN, S.W. 1955. Canine paragonimiasis. North American Veterinarian 36: 657-662.

NIILO, L. 1968. Bovine hemorrhagic filariasis in cattle imported into Canada. Canadian Veterinary Journal 9: 132-137.

NOWAK, R.M., AND J.L. PARADISO. 1983. Walker's Mammals of the World. 4th Edition, Vols. I and II. The Johns Hopkins University Press. Baltimore. 1362 pp. + XXV.

O'DONOGHUE, J.G. 1958. Clinical trials with Cyanacethydrazide for the treatment of lungworms in cattle and sheep. Canadian Journal of Comparative Medicine 22: 237-239.

OSLER, W. 1877. Verminous bronchitis in dogs. Veterinarian London 50: 387-397.

OSLER, W., AND A.W. CLEMENT. 1883. An investigation into the parasites in the pork supply of Montreal. 14 pp. Montreal Gazette Printing Co. 1883.

PALLISTER, E., AND P.J.G. PLUMMER. 1941. Multicystic hydatid in the liver of a hog. Canadian Journal of Comparative Medicine 5: 291-292.

POLLEY, L.R., P.E. MOSTERT. 1980. Ascaris suum in Saskatchewan pigs: an abattoir survey of prevalence and intensity of infection. Canadian Veterinary Journal 21: 307-309.

RANSOM, B.H., AND S. HADWEN. 1918. Horse strongyles in Canada. Journal of the American Veterinary Medical Association 6: 202-214.

RICH, G.B. 1952. A review of studies on the systemic control of livestock insect parasites. Proceedings of the B.C. Entomological Society 48: 58-59.

1957. The ear tick, Otobius megnini (Duges) (Acarina:
Argasidae), and its record in British Columbia. Canadian Journal of Comparative Medicine 21: 415-418.

SANFORD, S.E., and G.K.A. JOSEPHSON. 1982. Bovine cryptosporidiosis: Clinical and pathological findings in forty-two scouring neonatal calves. Canadian Veterinary Journal 23: 343-347.

SAUNDERS, L.G. 1949. A survey of helminth and protozoan incidence in man and dogs at Fort Chipewyan, Alberta. Journal of Parasitology 35: 31-34.

SAUVAGEAU, R., AND J.L. FRECHETTE. 1980. Ascaridiose hepatique chez un agneau. Canadian Veterinary Journal 21: 66.

SCHMIDT, G.D. 1986. CRC Handbook of Tapeworm Identification. $688 \mathrm{pp}$.

SCHOLTEN, T.H., K. RONALD, and D.M. MCLEAN. 1962. Parasite fauna of the Manitoulin Island Region. Canadian Journal of Zoology 40: 605-606.

SEAH, S.K.K., G. HUCAL, AND C. LAW. 1975. Dogs and intestinal parasites: a public health problem. Canadian Medical Association Journal 112: 1191-1194.

SLOCOMBE, J.O.D. 1970. Ostertagia trifurcata (Nematoda: Trichostrongylidae) in cattle in Canada. Canadian Journal of Zoology 48: 1143-1144.

1973a. Parasitisms in domesticated animals in Ontario. I. Ontario Veterinary College Records 1965-1970. Canadian Veterinary Journal 14: 36-42.

1973b. Gastrointestinal parasites in cattle in Ontario. Canadian Veterinary Journal 14: 91-95.

1973c. Morphological types in Haemonchus contortus (Nematoda) populations in Ontario sheep. Canadian Journal of Zoology 51: 1161-1163.

1974. Abomasal nematodes in cattle in Ontario. Canadian Journal of Comparative Medicine 38: 18-21.

SLOCOMBE, J.O.D., AND B.M. McCRAW. 1973. Gastrointestinal nematodes in horses in Ontario. Canadian Veterinary Journal 14: 101-105.

SMITH, F.R., AND W. THRELFALL. 1973. Helminths of some mammals from Newfoundland. American Midland Naturalist 90: 215-218.

SMITH, H.J. 1961. Bovine demodicidosis. I. Incidence in Ontario. Canadian Journal of Comparative Medicine 25: 165-169.

1970. On the development of gastrointestinal parasitisms in bovine yearlings. Canadian Journal of Comparative Medicine 34: 303-308.

1976a. Strongyle infections in ponies. I. Response to intermittent thiabendazole treatments. Canadian Journal of Comparative Medicine 40: 327-333.

1976b. Strongyle infections in ponies. II. Reinfection of treated animals. Canadian Journal of Comparative Medicine 40: 334-340.

1979a. Transmission of Oesophagostomum species in swine on pasture in the Maritime provinces. Canadian Veterinary Journal 20: 184-185. 
1979b. Probstmayria vivipara pinworms in ponies. Canadian Journal of Comparative Medicine 43: 341-342.

SMITH, H.J., AND R. McG. ARCHIBALD. 1965. The overwinter survival of ovine gastrointestinal parasites in the Maritime Provinces. Canadian Veterinary Journal 6: 257-267

1968. The effects of age and previous infection on the development of gastrointestinal parasitisms in cattle. Canadian Journal of Comparative Medicine 32: 511-517.

SMITH, H.J., A.B. HAWKES. 1978. Kidney worm infection in feral pigs in Canada with transmission to domestic swine. Canadian Veterinary Journal 19: 40-43.

SMITH, H.J., AND J.P. PERREAULT. 1972. A type II ostertagiasis outbreak in cattle in New Brunswick. Canadian Veterinary Journal 13: 114-117.

SOEHL, H. 1984. Cysticercus ovis in Nova Scotia. Communication, Food Production and Inspection Branch, Agriculture Canada 2: 7-9.

SPENCER, G.J. 1936. A check list of the fleas of British Columbia with a note on fleas in relation to sawdust in homes. Proceedings of the B.C. Entomological Society 32: 11-17.

1938. Ectoparasites of birds and mammals of British Columbia. II. A preliminary list of the Pupipara, louse flies. Proceedings of the B.C. Entomological Society 34: 39-45.

1939. Ectoparasites of birds and mammals in British Columbia. V. Parasites of domestic animals (Mammals). Proceedings of the B.C. Entomological Society 35: 19-23.

1940. Ectoparasites of birds and mammals in British Columbia. VI. A preliminary list of parasitic mites. Proceedings of the B.C. Entomological Society (1940): 14-18.

1960. The brown dog-tick, Rhipicephalus sanguineus (Latr.) in Vancouver. Proceedings of the B.C. Entomological Society 57: 64-65.

1966. Anoplura from British Columbia and some adjacent areas. Journal of the Entomological Society of British Columbia 63: 23-30.

STOCKDALE, P.H.G., G.K. ASHTON, M.A. HOWES, AND E. EWERT. 1973. Hyostrongylosis in Ontario. Canadian Veterinary Journal 14: 265-268.

STOCKDALE, P.H.G., AND J.P. LAUTENSLAGER. 1973. Unusual gastric nematodes of swine in Ontario. Canadian Veterinary Journal 14: 215-216.

SWALES, W.E. 1933a. A Review of Canadian Helminthology. I. The present status of knowledge of the helminth parasites of domesticated and semidomesticated mammals and economically important birds in Canada, as determined from work published prior to 1933. Canadian Journal of Research 8: 468-477.

1933b. A review of Canadian Helminthology. II.
Additions to part I, as determined from a study of parasitic helminths collected in Canada. Canadian Journal of Research 8: 478-482.

1939. Tests of Phenothiazine, a highly efficient anthelmintic. On a means of administration and the indicated uses for the control of parasitic diseases of sheep. Canadian Journal of Comparative Medicine 3: 188-194.

1940a. The helminth parasites and parasitic diseases of sheep in Canada. I. A survey and some preliminary studies on existing problems. Canadian Journal of Research 18: 29-48.

1940b. The helminth parasites and parasitic diseases of sheep in Canada. II. Notes on the effect of winter upon the free-living stages of nematode parasites of sheep on the pastures in Eastern Canada. Canadian Journal of Comparative Medicine and Veterinary Science 4: 155-161.

SWALES, W.E., AND L. CHOQUETTE. 1940. Diagnostic et traitement des maladies parasitaires du mouton dans la province de Quebec. Canadian Journal of Comparative Medicine 4: 292-294.

SWALES, W.E., P.E. SYLVESTRE, AND S.B. WILLIAMS. 1942. Field trials of control measures for parasitic diseases of sheep. Canadian Journal of Research 20: 115-132.

SWEATMAN, G.K. 1958. Biology of Otodectes cynotis, the ear canker mite of carnivores. Canadian Journal of Zoology 36: 849-862.

SWEATMAN, G.K., AND P.J.G. PLUMMER. 1957. The biology and pathology of the tapeworm Taenia hydatigena in domestic and wild hosts. Canadian Journal of Zoology 35: 93-109.

TAILYOUR, J.M., AND J.R. STEELE. 1960. Trichinella infection in swine in the Vancouver area. Canadian Journal of Public Health 51: 309.

TESKEY, H.J. 1960. Survey of insects affecting livestock in southwestern Ontario. Canadian Entomologist 92: 531-544.

THOMPSON, G.B. 1934. Records of Siphunculata and Mallophaga from Canadian hosts. Canadian Entomologist 66: 279-281

THRELFALL, W. 1969. Further records of helminths from Newfoundland mammals. Canadian Journal of Zoology 47: 197-201.

TYRRELL, J.B. 1883. On the occurrence in Canada of two species of parasitic mites. Proceedings of the Canadian Institute 1: 332-342.

UNRUH, D.H.A., J.E. KING, R.D.P. EATON, AND J.R. ALLEN. 1973. Parasites of dogs from Indian settlements in northwestern Canada: A survey with Public Health Implications. Canadian Journal of Comparative Medicine 37: 25-32.

WARDLE, R.A., AND E.L. McCOLL. 1937. The taxonomy of Diphyllobothrium latum (Linne, 1758) in Western Canada. Canadian Journal of Research 15: 163-175. 
WATSON, E.A., AND S. HADWEN. 1912.

Trypanosomes found in Canadian mammals.

Parasitology 5: 21-26.

WATSON, T.G. 1981. Growth of Metorchis conjunctus (Cobbold, 1860) Looss, 1899 (Trematoda:

Opisthorchiidae) in the bile ducts of various definitive hosts. Canadian Journal of Zoology 59: 2014-2019.

WEBSTER, G.A. 1958. On prenatal infection and the migration of Toxocara canis Werner, 1782, in dogs. Canadian Journal of Zoology 36: 435-440.

WEBSTER, W.A., and D.B. WILKINS. 1970. The recovery of Parafilaria bovicola Tubangui, 1934 from an imported Charolais bull. Canadian Veterinary Journal 11: 13-14.

WEBSTER, W.A., T.W. DUKES, AND A. BUNDZA. 1977. Onchocerciasis (Nematoda: Filariodea) in bovine leg joints. Canadian Journal of Zoology 55: 1067-1070.

WEBSTER, W.A., AND T.W. DUKES. 1979. Bovine and equine onchocerciasis in eastern North America with a discussion on cuticular morphology of Onchocerca sp. in cattle. Canadian Journal of Comparative Medicine 43: $330-332$

WEBSTER, W.A., R.R. MacKAY. 1969. Two new host records for Nematodirus species. Canadian Journal of Zoology 47: 1411.

WEINTRAUB, J. 1952. A preliminary report on acaricides tested for protection against the tick Dermacentor andersoni Stiles on cattle. Proceedings of the B.C. Entomological Society 48: 51-54.

WHITEHEAD, W.E. 1934. Records of some Quebec Mallophaga and Anoplura. Report of the Quebec Society for the Protection of Plants 26: 84-87.
WILKINSON, P.R. 1968. Phenology, behavior, and hostrelations of Dermacentor andersoni Stiles in outdoor "rodentaria," and in nature. Canadian Journal of Zoology 46: 677-689.

1972. Sites of attachment of 'prairie' and 'montane' Dermacentor andersoni (Acarina: Ixodidae) on cattle. Journal of Medical Entomology 9: 133-137.

1981. The proportion of immature stages of the Rocky Mountain wood tick (Dermacentor andersoni) feeding on artificially infested cattle. Journal of the Entomological Society of B.C. 78: 38-42.

1982. The potential for selection of cattle resistant to tick paralysis and attachment of Rocky Mountain wood ticks. Agriculture Canada, Research Highlights 1981 pp. 25-28, Lethbridge, Alberta.

WILKINSON, P.R., AND J.E. LAWSON. 1965. Differences of sites of attachment of Dermacentor andersoni Stiles to cattle in southeastern Alberta and in south central British Columbia, in relation to possible existence of genetically different strains of ticks. Canadian Journal of Zoology 43: 408-411.

WITTNICH, C. 1976. Entamoeba histolytica infections in a german shepherd dog. Canadian Veterinary Journal 17: $259-263$.

WOBESER, G.A. 1971. The occurrence of Echinococcus multilocularis (Leuckart, 1863) in cats near Saskatoon, Saskatchewan. Canadian Veterinary Journal 12: 65-68.

WOO, P., M.A. SOLTYS, AND A.C. GILLICK. 1970. Trypanosomes in cattle in Southern Ontario. Canadian Journal of Comparative Medicine 34: 142-147.

WRIGHT, B. 1979. Mites, ticks, fleas, and lice in the Nova Scotia Museum and Acadia University Museum collections. Science 29: 185-196.

\section{Supplementary Readings}

ALLEN, J.A., AND A.B. WICKWARE. 1922. A preliminary note on parasites infesting domesticated silver black foxes in Canada. Parasitology 14: 27-28.

ANON. 1931. A note on Parametorchis canadensis (Law) from Ontario mink. Report of the Ontario Veterinary College for 1930: 39 .

1948. Liver fluke control in the Burwash area. Report of the Ontario Veterinary College (for 1947): 49-51.

1950. Survey to ascertain the geographical distribution of the large American liver fluke (Fascioloides magna) in Ontario. Report of the Ontario Veterinary College for 1949: 35 .

ARTHUR, D.R., AND K.R. SNOW. 1968. Ixodes pacificus Cooley and Kohls, 1943: Its life-history and occurrence. Parasitology 58: 893-906.

BATT, H.E. 1926. Internal parasites in foxes. Fur Trade Journal of Canada 3: 7 .

BECKLUND, W.W. 1964. Revised check list of internal and external parasites of domestic animals in the United States and possessions and in Canada. American
Journal of Veterinary Research 25: 1380-1446.

BROWN, J.H. 1955. Colorado tick fever in Alberta. Canadian Journal of Zoology 33: 389-390.

BRUCE, E.A. 1920. Information for farmers and ranchers regarding tick paralysis in British Columbia. Dominion of Canada, Department of Agriculture, Bulletin No. 133. $7 \mathrm{pp}$.

CAMERON, A.E. 1923. Notes on buffalo: anatomy. pathological conditions, and parasites. The Veterinary Journal 79: 331-336.

1924. Some further notes on buffalo. The Veterinary Journal 80: 413-417.

CAMERON, T.W.M. 1933. The important helminth parasites of stock in the British Empire. British Veterinary Journal 89: 505-514.

1944. The morphology, taxonomy, and life history of Metorchis conjunctus (Cobbold, 1860). Canadian Journal of Research 22: 6-16.

CAMPBELL, D.J. 1960. Parasitic gastro-enteritis of sheep. Canadian Veterinary Journal 1: 151-156. 
COGLEY, T.P., J.R. ANDERSON, AND J.

WEINTRAUB. 1981. Ultrastructure and function of the attachment organ of warble fly eggs (Diptera: Oestridae, Hypodermatinae). International Journal of Insect Morphology and Embryology 10: 7-18.

CURTIS, L.C. 1959. Control of the Rocky Mountain wood tick, Dermacentor andersoni Stiles (Acarina: Ixodidae), with ground sprays of Dieldrin and Heptachlor. Proceedings of the B.C. Entomological Society 56: 13-15.

FRIEND, S.C.E., and P.H.G. STOCKDALE. 1980. Experimental Eimeria bovis infection in calves: a histopathological study. Canadian Journal of Comparative Medicine: 44: 129-140

GADBOIS, P., J.L. FRECHETTE, A. VILLENEUVE, AND B.I. GROVES. 1985. A new approach in the prevention of gastrointestinal parasitic infections in cattle. Canadian Veterinary Journal 26: 127-131.

GAJADHAR, A.A., J.P. CARON, AND J.R. ALLEN. 1985. Cryptosporidiosis in two foals. Canadian Veterinary Journal 26: 132-134.

GOODING, R.H., AND J. WEINTRAUB. 1960. The genitalia of Hypoderma bovis (L.) and H. lineatum (De Vill.) (Diptera: Oestridae). Canadian Journal of Zoology 38: 565-574.

GREGSON, J.D. 1936. Studies on the rate of tick feeding in relation to disease. Proceedings of the B.C. Entomological Society (1936): 15-21.

1940. The discovery of an ixovotoxin in Dermacentor andersoni eggs. Proceedings of the B.C. Entomological Society (1940): 9-10.

1942a. The coast tick (Ixodes californicus Banks) problem in British Columbia. Canadian Entomologist 74: 3-5.

1942b. Notes on the laboratory rearing of some Canadian ticks. Proceedings of the B.C. Entomological Society (1942): 32-35.

1943. The enigma of tick paralysis. Proceedings of the

B.C. Entomological Society (1943) 40: 19-23.

1951. Notes on the spring activity of the Rocky Mountain wood tick, Dermacentor andersoni Stiles (Acarini: Ixodidae). Proceedings of the B.C. Entomological Society 47: 4-7.

GREGSON, J.D., AND G.P. HOLLAND. 1944. Devices for charting and obtaining naturally emerged cattle warbles (Diptera: Oestridae). Proceedings of the B.C. Entomological Society (1944) 41: 31-33.

HADWEN, S. 1914. Notes on the life-histories of bloodsucking Diptera of British Columbia, with special reference to the Tabanidae. Proceedings of the B.C. Entomological Society (1914): 46-49.

HEARLE, E. 1938. Insects and allied parasites injurious to livestock and poultry in Canada. Canadian Department of Agriculture Publicate 604 (Farmers Bulletin 53).

HOLLAND, G.P. 1939. Notes on the ecology of Dermacentor andersoni in southern Alberta.
Proceedings of the B.C. Entomological Society (1939) 8-11.

KENNEDY, A.H. 1950. Paragonimus infection in a ranchraised fox. Report of the Ontario Veterinary College for 1949: 132-137.

KINGSCOTE, A.A. 1930. A case of paragonimiasis in the Ontario domestic fox. Report of the Ontario Veterinary College, p. 40.

1932. A case of Paragonimus kellicotti (lung-fluke) infestation in ranch-raised mink. Report of the Ontario Veterinary College for 1931: 62 .

1938. Common parasites of Canadian animals. Canadian Journal of Comparative Medicine 2: 47-55. 1953. Internal parasites of swine. Ontario Department of Agriculture Bulletin No. 496. 36 pp. Toronto, Canada.

LAW, R.G. 1930. The mink in captivity. Ontario Department of Game Fish Bulletin 3: 1-23 (see p. 12-18).

LAW, R.G., AND A.C. SECORD. 1931. Control of lung worm in silver foxes. North American Veterinarian 12: $42-44$.

LINDQUIST, E.E. 1979. 12. Acari pp. 252-290. In H.V. Danks (ed.). Canada and its Insect Fauna. Memoirs of the Entomological Society of Canada 108.573 pp.

LUBINSKY, G. 1958. On the 12-hooked oncospheres of Canadian strains of Echinococcus. Canadian Journal of Zoology 36: 883-887.

MAIL, G.A. 1942. Tick control with special reference to Dermacentor andersoni Stiles. Canadian Journal of Agriculture Science 23: 59-67.

MARSH, H. 1952. Disease problems in range livestock. Canadian Journal of Comparative Medicine 16: 89-96.

McALLISTER, R.A. 1964. An outbreak of Toxoplasmosis in an Ontario chinchilla herd. Canadian Journal of Comparative Medicine 28: 53-56.

McCRAW, B.M. 1951. Some aquatic snails of Ontario and their relation to the possible occurrence of trematode diseases: a preliminary report. Report of the Ontario Veterinary College for 1950: 128-139.

McGREGOR, J.K., AND D.C. MAPLESDEN. 1955. Gastro-intestinal helminthiasis of sheep. A commentary and case report. Canadian Journal of Comparative Medicine 19: 129-131.

MOILLIET, T.K. 1936. A review of tick paralysis in cattle in British Columbia with mites in several cases. Proceedings of the B.C. Entomological Society (1936): 35-39.

1937. A review of tick paralysis in cattle in British Columbia. Proceedings of the B.C. Entomological Society 33: 35-39.

NELSON, W.A., AND J. WEINTRAUB. 1972. Hypoderma lineatum (De Vill.) (Diptera: Oestridae): invasion of the bovine skin by newly hatched larvae. Journal of Parasitology 58: 614-624.

NIILO, T. 1970. Bovine coccidiosis in Canada. Canadian Veterinary Journal 11: 91-98. 
OLIVER, J.H. JR., P.R. WILKINSON, AND G.M. KOHLS. 1972. Observations on hybridization of three species of North American Dermacentor ticks. Journal of Parasitology 58: 380-384.

PARNELL, I.W. 1934. Studies on the bionomics and control of the bursate nematodes of horses and sheep. I. The effect of urine on the eggs and larvae in the feces. Canadian Journal of Research: 532-538.

PULLIN, J.W. 1961. A discussion of the common diseases of sheep. Canadian Veterinary Journal 2: 359-368.

RAPSEY, G.H., AND R.G. LAW. 1928. Liver fluke in Mink. The Black Fox Magazine 12: 10.

SCHILLHORN VAN VEEN, T.W. 1982. Fasciola cercariae in Quebec. Canadian Veterinary Journal 23: 163.

SLOCOMBE, J.O.D. 1978. Heartworm in dogs in Canada in 1977. Canadian Veterinary Journal 19: 244-247.

SLOCOMBE, J.O.D., AND I. McMILLAN. 1979. Heartworm in dogs in Canada in 1978. Canadian Veterinary Journal 20: 284-289.

1980. Heartworm in dogs in Canada in 1979. Canadian Veterinary Journal 21: 159-161.

1981. Heartworm in dogs in Canada in 1980. Canadian Veterinary Journal 22: 201-203.

1982. Heartworm in dogs in Canada in 1981. Canadian Veterinary Journal 23: 219-221.

1983. Heartworm in dogs in Canada in 1982. Canadian Veterinary Journal 24: 227-229.

1984. Heartworm in dogs in Canada in 1984. Canadian Veterinary Journal 26: 323-327.

SPENCER, G.J. 1952. The 1951 status knowledge of the insects of British Columbia. Proceedings of the B.C. Entomological Society 48: 36-41.

1958. On the reproductive potential of the sheep nostril fly Oestrus ovis L. (Diptera: Oestridae). Proceedings of the B.C. Entomological Society 55: 25-26.

STOCKDALE, P.H.G. 1977. The pathogenesis of the lesions produced by Eimeria zuernii in calves. Canadian Journal of Comparative Medicine 41: 338-344.

STOCKDALE, P.H.G., A.R. BAINBOROUGH, C.B. BAILEY, and L. NIILO. 1981. Some pathophysiological changes associated with infection of Eimeria zuernii in calves. Canadian Journal of Comparative Medicine 45: 34-37.

STOCKDALE, P.H.G., and L. NIILO. 1976. Production of bovine coccidiosis with Eimeria zuernii. Canadian Veterinary Journal 17: 35-37.

STRICKLAND, E.H. 1949. Wohlfahrtia (Diptera, Metopiidae) myiasis of mink in Alberta. Canadian Entomologist 81: 58-60.

SWALES, W.E. 1939. Notes on the diagnosis and treatment of parasitic diseases of sheep in Canada. Canadian Journal of Comparative Medicine 3: 341-344. 1940. Further experiments on the use of phenothiazine as an anthelmintic for sheep. Canadian Journal of Research 18: 266-271.

SWEATMAN, G.K., AND T.C. HENSHALL. 1962. The comparative biology and morphology of Taenia ovis and Taenia krabbei, with observations on the development of $T$. ovis in domestic animals. Canadian Journal of Zoology 40: 1287-1311.

TAILYOUR, J.M., AND M.J. HAMPTON. 1954. A check on the incidence of trichinosis in swine on six piggeries in British Columbia. Canadian Journal of Comparative Medicine 18: 311-312.

TYRRELL, J.B. 1883. On the occurrence in Canada of two species of parasitic mites. Proceedings of the Canadian Institute 1: 332-342.

WEBSTER, G.A. 1958. A report on Toxocara canis Werner, 1782. Canadian Journal of Comparative Medicine 22: 272-279.

WHITNEY, H., A. VILLENEUVE, J.L. FRECHETTE, AND B. PINEL-ALLOUL. 1981. Intermediary hosts for Fasciola hepatica identified in Quebec. Canadian Veterinary Journal 22: 334.

WICKWARE, A.B. 1923. Parasites of the silver-black fox. Canadian Veterinary Record 4: 128-129.

WILKINSON, P.R. 1967. The distribution of Dermacentor ticks in Canada in relation to bioclimatic zones. Canadian Journal of Zoology 45: 517-537.

WILLSON, P.J., and S.D. ACRES. 1982. A comparison of dichromate solution floatation and fecal smears for diagnosis of cryptosporidiosis in calves. Canadian Veterinary Journal 23: 240-246.

WOOLFGANG, R.W., AND J.B. POOLE. 1956. Distribution of Echinococcus disease in Northwestern Canada. American Journal of Tropical Medicine and Hygiene 5: 869-871.

YANG, Y.H., AND H.C. GRICE. 1964. Klossiella muris parasitism in laboratory mice. Canadian Journal of Comparative Medicine 28: 63-66. 
Host Index

Artiodactyla 23

Bos taurus 23

Bovidae 23

Canidae 25

Caprahircus 24

Carnivora 25

Cat 26

Dog 25

Equidae 27

Equus caballus

Eutheria 23
Canis familiaris 25

\begin{tabular}{lll} 
Felidae 26 & \multicolumn{2}{c}{26} \\
Felis catus & 26 \\
Goat 24 & \\
Horse 27 & \\
Mammalia & 23 \\
Ovis aries & 24 & \\
Ox 23 & & \\
Perissodactyla & 27 \\
Pig 25 & \\
Sheep 24 & \\
Suidae 25 & \\
Sus scrofa 25 & 25
\end{tabular}

\section{Parasite Index}

Acanthocephala

17

Acari 18

Acaridida 18

Acariformes 18

Actinedida 18

Adenophorea 7

Aelurostrongylus spp. $\quad 12$

Alaria arisaemoides 4

Alaria canis 4

Alaria spp. 4

Alfortia edentatus

see Strongylus edentatus

14

Alfortia spp.

see Strongylus spp. $\quad 14$

Amoebida 3

Ancylostomatidae 11

Ancylostomatidae (unidentified)

11

Ancylostomatoidea 11

Ancylostomum caninum

11

Ancylostomum spp. 11

Angiostrongylidae $\quad 12$

Animalia 1

Anoplocephala magna 5

Anoplocephala mamillana

see Paranoplocephala mamillana 5

Anoplocephala perfoliata 5

Anoplocephala spp. 5

Anoplocephalidae 5

Apicomplexa 1

Arachnida 18

Archiacanthocephala

Argasidae $\quad 18$

Ascaridida 8

Ascarididae 8

Ascaridoidea 8

Ascaris lumbricoides

Ascaris suum 8

Ascaris spp. $\quad 8$

Ascarops spp. 11

Atractidae 9

Balantidium spp.

Belascaris mystax see Toxocara cati 9

Bovicola bovis

see Damalinia bovis 21

Bovicola caprae

see Damalinia caprae 21

Bunostomum phlebotomum

Bunostomum trigonocephalum

Bunostomum spp. 11

Calicophoron microbothrioides

Calliphora spp. 20

Calliphoridae 20

Calliphoridae (unidentified)

Capillaria aerophila 7

Capillaria hepatica 7

Capillaria longipes 7

Capillaria spp. 7

Ceratophyllidae 21

Cestoidea 5

Cestoidea gen. spp. 7

Chabertia ovina 12

Chabertia spp. $\quad 12$

Chabertiidae 12

Chelicerata 18

Cheyletidae 18

Cheyletiella spp. $\quad 18$

Chorioptes bovis 18

Ciliophora 2

Citellinema bifurcatum $\quad 15$

Cooperia curticei 16

Cooperia oncophora 16

Cooperia punctata 16

Cooperia spp. 16

Corynosoma semerme

Cosmocercoidea 9

Crenosoma vulpis $\quad 12$

Crenosomatidae 12

Cryptocotyle lingua 4

Cryptosporidium spp. 1

Ctenocephalides canis $\quad 21$

Ctenocephalides felis $\quad 21$ 
Ctenocephalides felis felis

Ctenocephalides spp.

Cuterebra spp. $\quad 20$

Cuterebridae 20

Cyathostoma spp. 15

Cyathostomum catinatum

Cyathostomum coronatum 13

Cyathostomum labiatum 13

Cyathostomum labratum $\quad 13$

Cyclophyllidea 5

Cylicocyclus elongatus 13

Cylicocyclus insigne 13

Cylicocyclus nassatus $\quad 13$

Cylicocyclus radiatus $\quad 13$

Cylicodontophorus bicornatus

Cylicostephanus calicatus 13

Cylicostephanus goldi 14

Cylicostephanus poculatus $\quad 14$

Cylicostomum bicornatum

see Cylicodontophorus bicornatus 13

Cylicostomum calicatum see Cylicostephanus calicatus 13

Cylicostomum catinatum see Cyathostomum catinatum 13

Cylicostomum coronatum see Cyathostomum coronatum 13

Cylicostomum elongatum see Cylicocyclus elongatus 13

Cylicostomum goldi see Cylicostephanus goldi 14

Cylicostomum insigne see Cylicocyclus insigne 13

Cylicostomum labiatum see Cyathostomum labiatum 13

Cylicostomum labratum see Cyathostomum labratum 13

Cylicostomum nassatum see Cylicocyclus nassatus 13

Cylicostomum poculatum see Cylicostephanus poculatus 14

Cylicostomum radiatum see Cylicocyclus radiatus $\quad 13$

Cysticercus bovis 6

Cysticercus cellulosae 6

Cysticercus ovis 6

Cysticercus tenuicollis 6

Damalinia bovis 21

Damalinia caprae 21

Damalinia equi 21

Damalinia limbata 21

Damalinia spp. 21

Delafondia vulgaris see Strongylus vulgaris 14

Demodex bovis 18

Demodex phylloides

Demodex spp. $\quad 18$

Demodicidae 18

Dermacentor albipictus 19

Dermacentor andersoni 19

Dermacentor erraticus var. albipictus see Dermacentor albipictus 19
Dermacentor variabilis

Dermacentor venustus see Dermacentor andersoni

Dermacentor spp. $\quad 19$

Dermanyssidae 18

Dicrocoeliidae 4

Dicrocoelium dendriticum

Dicrocoelium lanceolatum see Dicrocoelium dendriticum

Dictyocaulidae $\quad 15$

Dictyocaulus arnfieldi $\quad 15$

Dictyocaulus filaria 15

Dictyocaulus viviparus $\quad 15$

Dictyocaulus spp. $\quad 15$

Digenea 4

Digenea gen. spp. 5

Dilepididae 5

Dioctophyma renale 7

Dioctophymatidae 7

Dioctophymatoidea 7

Dipetalonema spp. 10

Diphyllobothriidae 7

Diphyllobothrium dendriticum

Diphyllobothrium latum 7

Diphyllobothrium spp. 7

Diplomonadida 3

Diplostomidae 4

Diptera 20

Diptera (unidentified) 21

Dipylidium caninum 5

Dipylidium spp. 6

Dirofilaria immitis $\quad 10$

Dirofilaria spp. $\quad 10$

Dracunculidae $\quad 10$

Dracunculoidea 10

Dracunculus insignis $\quad 10$

Echinococcus granulosus 6

Echinococcus multilocularis 6

Echinococcus spp. 6

Eimeria ahsata $\quad 1$

Eimeria alabamensis 1

Eimeria arloingi 1

Eimeria aubernensis 1

Eimeria bovis 1

Eimeria bukidnonensis 1

Eimeria canadensis 1

Eimeria canis 1

Eimeria crandallis 1

Eimeria cylindrica 1

Eimeria dublieki 1

Eimeria ellipsoidalis 1

Eimeria faurei 1

Eimeria granulosa 1

Eimeria intricata 1

Eimeria leuckarti 2

Eimeria ninakohlyakimovae

Eimeria pallida 2

Eimeria parva 2

Eimeria punctata 2

Eimeria wyomingensis 2

Eimeria spp. 2 
Eimeriina 1

Endolimax nana 3

Enoplida 7

Entamoeba blattae 3

Entamoeba coli 3

Entamoeba histolytica 3

Eucestoda 5

Eucoccidiida 1

Eucoccidiida (unidentified) 2

Eumetazoa 4

Fasciola hepatica 4

Fasciolidae 4

Fascioloides magna 4

Felicola subrostratus $\quad 21$

Filarioidea 10

Filaroides milksi 12

Filaroides spp.

Filariidae 10

Filaroididae $\quad 12$

Gamasida $\quad 18$

Gasterophilidae $\quad 20$

Gasterophilus intestinalis

Gasterophilus nasalis $\quad 20$

Gasterophilus spp.

Giardia canis 3

Giardia spp. 3

Gongylonema pulchrum

Gongylonematidae 11

Gyalocephalus capitatus

Gyalocephalus spp. $\quad 14$

Habronema majus $\quad 10$

Habronema microstoma see Habronema majus

Habronema muscae 10

Habronema spp. $\quad 10$

Habronematidae $\quad 10$

Habronematoidea 10

Haemaphysalis cinnabarina

Haemaphysalis leporis-palustris

Haematopinidae 22

Haematopinus asini $\quad 22$

Haematopinus eurysternus

Haematopinus suis 22

Haematopinus spp. 22

Haemonchus contortus

Haemonchus spp. $\quad 16$

Heligmosomidae $\quad 15$

Heterophyidae 4

Hexamitidae 3

Hippobosidae 20

Hyostrongylus rubidus $\quad 16$

Hyostrongylus spp. 16

Hypoderma bovis 20

Hypoderma lineatum 20

Hypoderma spp. 20

Hyostrongylus rubidus

Hyostrongylus spp. $\quad 16$

Hystrichopsyllidae

Insecta 20
Ischnocera 21

Isospora bigemina 2

Isospora felis 2

Isospora rivolta 2

Isospora spp. 2

Ixodes cookei 19

Ixodes hexagonus var. cookei see Ixodes cookei

Ixodes ricinus 19

Ixodes ricinus var. scapularis seeIxodes spp. $\quad 19$

Ixodida 18

Ixodidae 19

Kinetofragminophorea 2

Kinetoplastida 3

Klossiella equi 1

Lice (unidentified) 22

Linognathidae 22

Linognathus pedalis $\quad 22$

Linognathus piliferus see Linognathus setosus 22

Linognathus setosus $\quad 22$

Linognathus stenopsis $\quad 22$

Linognathus vituli 22

Linognathus spp. $\quad 22$

Lobosea 3

Lucilia illustris $\quad 20$

Lucilia sericata 20

Macracanthorhynchus hirudinaceus

Mallophaga 21

Mastigophora 3

Melophagus ovinus $\quad 20$

Metastrongylidae $\quad 12$

Metastrongyloidea $\quad 12$

Metastrongylus elongatus see Metastrongylus salmi 12

Metastrongylus pudendotectus 12

Metastrongylus salmi 12

Metastrongylus spp. $\quad 12$

Metorchis conjunctus 4

Metorchis spp. 4

Molineidae 15

Moniezia benedeni 5

Moniezia expansa 5

Moniezia spp. 5

Monodontus phlebotomus

see Bunostomum phlebotomum 11

Monodontus trigonocephalus

see Bunostomum trigonocephalum 11

Monodontus spp.

see Bunostomum spp.

Monopsyllus vison 21

Muellerius capillaris $\quad 12$

Muellerius spp. $\quad 12$

Multiceps spp. 6

Nemathelminthes 7

Nematoda 7

Nematoda (unidentified) 
Nematodirus abnormalis

Nematodirus filicollis

15

Nematodirus helvetianus 15

Nematodirus spathiger

Nematodirus spp. $\quad 16$

Notoedres spp. $\quad 18$

Oesophagodontus robustus

14

Oesophagodontus spp. 14

Oesophagostomum columbianum

Oesophagostomum dentatum

12

Oesophagostomum quadrispinulatum 12

Oesophagostomum radiatum 13

Oesophagostomum venulosum

Oesophagostomum spp.

13

Oestridae 20

Oestrus ovis 21

Oligacanthorhynchida $\quad 17$

Oligacanthorhynchidae $\quad 17$

Ollulanus tricuspis $\quad 16$

Onchocerca cervicalis $\quad 10$

Onchocerca gutturosa 10

Onchocerca lienalis 10

Onchocerca spp. 10

Onchocercidae $\quad 10$

Opisthorchidae 4

Oslerus osleri 12

Ostertagia circumcincta 16

Ostertagia lyrata $\quad 16$

Ostertagia ostertagi 17

Ostertagia trifurcata 17

Ostertagia spp. 17

Otobius lagophilus $\quad 18$

Otobius megnini 19

Otobius spp. $\quad 19$

Otodectes cynotis $\quad 18$

Otodectes cynotis var. canis $\quad 18$

Otodectes cynotis var. felis $\quad 18$

Oxyurida 9

Oxyuridae 9

Oxyuris equi 9

Oxyuris spp. 9

Oxyuroidea 9

Palaeacanthocephala $\quad 17$

Parafilaria bovicola 10

Paragonimus kellicotti 5

Paragonimus spp. 5

Parametorchis complexus 4

Parametorchis manitobensis see Parametorchis complexus

Paramphistomidae 4

Paramphistomum liorchis 4

Paramphistomum microbothrioides see Calicophoron microbothrioides 4

Paramphistomum spp. 4

Paranoplocephala mamillana 5

Parascaris equorum 8

Parascaris spp. 8

Parasitiformes 18

Parelaphostrongylus tenuis

Pelodera strongyloides 9
Petrovinema poculatum

see Cylicostephanus poculatus

Phaenicia sericata see Lucilia sericata $\quad 20$

Phormia regina 20

Phormia terranovae 20

Physaloptera rara 10

Physalopteridae $\quad 10$

Physalopteroidea $\quad 10$

Physocephalus spp. 11

Plagiorchiidae 5

Plagiorchis massino 5

Platyhelminthes 4

Pneumonyssus spp. $\quad 18$

Pneumostrongylus tenuis

see Parelaphostrongylus tenuis

Polymorphida 17

Polymorphidae $\quad 17$

Poteriostomum spp. $\quad 14$

Probstmayria vivipara 9

Protophormia terranovae see Phormia terranovae

Protostrongylidae $\quad 12$

Protostrongylus spp. $\quad 12$

Protozoa 1

Pseudophyllidea 7

Psoroptes spp. 18

Psoroptidae 18

Pulicidae 21

Rhabditida 9

Rhabditidae 9

Rhabditis strongyloides

see Pelodera strongyloides

Rhabditoidea 9

Rhabditoidea (unidentified) 9

Rhipicephalus sanguineus $\quad 19$

Rhipicephalus spp. $\quad 19$

Sarcocystis spp. 2

Sarcodina 3

Sarcomastigophora 3

Sarcoptes scabiei $\quad 18$

Sarcoptidae $\quad 18$

Secernentea 8

Setaria equina 10

Setaria labiatopapillosa $\quad 10$

Setaria spp. $\quad 10$

Siphonaptera 21

Siphunculata 22

Skrjabinema spp. 9

Solenopotes capillatus 22

Spirocerca spp. $\quad 11$

Spirocercidae 11

Spirurida 10

Spiruroidea 11

Sporozoea 1

Stephanofilaria stilesi $\quad 10$

Stephanurus dentatus $\quad 15$

Strongylida 11

Strongylidae 13

Strongylidae (unidentified) 
Strongyloidea 12

Strongyloides papillosus

Strongyloides stercoralis

Strongyloides spp. 9

Strongyloididae 9

Strongylus edentatus

Strongylus equinus

Strongylus vulgaris

Strongylus spp. 14

Syngamidae

15

Taenia crassiceps 6

Taenia elliptica 6

Taenia hydatigena 6

Taenia pisiformis 6

Taenia serialis 6

Taenia taeniaeformis 6

Taenia spp. 6

Taeniidae 6

Tamiophila grandis

Telodorsagia davtiani

Thelazia gulosa 11

Thelazia lacrymalis

Thelazia spp.

11

Thelaziidae 11

Thelazioidea 11

Thysanosoma actinioides

Thysanosoma spp. 5

Toxascaris leonina 8

Toxascaris limbata see Toxascaris leonina

Toxascaris spp. 8

Toxocara canis 9

Toxocara cati 9

Toxocara mystax see Toxocara cati 9

Toxocara spp. 9

Toxoplasma gondii 2

Toxoplasma spp. $\quad 2$

Trematoda 4

Trichinella spiralis $\quad 7$

Trichinellidae 7

Trichinelloidea 7

Trichodectes canis $\quad 21$

Trichodectes equi 21

Trichodectes spp. 21

Trichomonadida 3
Trichomonas spp. 3

Trichonema catinatum

see Cyathostomum catinatum 13

Trichostomatida 2

Trichostrongylidae $\quad 16$

Trichostrongyloidea $\quad 15$

Trichostrongyloidea (unidentified egg) 17

Trichostrongylus axei 17

Trichostrongylus colubriformis

Trichostrongylus extenuatus see Trichostrongylus axei 17

Trichostrongylus vitrinus

Trichostrongylus spp.

17

Trichuridae 7

Trichuridae (unidentified) $\quad 8$

Trichuris ovis 7

Trichuris suis 8

Trichuris vulpis 8

Trichuris spp. $\quad 8$

Triodontophorus brevicauda

Triodontophorus intermedius see Triodontophorus serratus

Triodontophorus minor 14

Triodontophorus serratus 14

Triodontophorus tenuicollis

Triodontophorus spp.

Troglotrematidae 5

Trombiculidae $\quad 18$

Trombiculidae (unidentified) $\quad 18$

Trypanosoma equiperdum 3

Trypanosoma rutherfordi 3

Trypanosoma theileri 3

Trypanosoma uniforme 3

Trypanosoma spp. 3

Uncinaria stenocephala 11

Uncinaria spp. $\quad 11$

Uniramia 20

Warrenius bifurcatus see Citellinema bifurcatum 15

Wohlfahrtia opaca 20

Wohlfahrtia vigil 20

Zoomastigophorea 3 

